\title{
LIPSCHITZ STABILITY IN THE DETERMINATION OF THE PRINCIPAL PART OF A PARABOLIC EQUATION
}

\author{
GanghuA YUAN ${ }^{1,2}$ And Masahiro YAMAMOto ${ }^{1}$
}

Abstract. Let $y(h)(t, x)$ be one solution to

$$
\partial_{t} y(t, x)-\sum_{i, j=1}^{n} \partial_{j}\left(a_{i j}(x) \partial_{i} y(t, x)\right)=h(t, x), 0<t<T, x \in \Omega
$$

with a non-homogeneous term $h$, and $\left.y\right|_{(0, T) \times \partial \Omega}=0$, where $\Omega \subset \mathbb{R}^{n}$ is a bounded domain. We discuss an inverse problem of determining $n(n+1) / 2$ unknown functions $a_{i j}$ by $\left\{\left.\partial_{\nu} y\left(h_{\ell}\right)\right|_{(0, T) \times \Gamma_{0}}\right.$, $\left.y\left(h_{\ell}\right)(\theta, \cdot)\right\}_{1 \leq \ell \leq \ell_{0}}$ after selecting input sources $h_{1}, \ldots, h_{\ell_{0}}$ suitably, where $\Gamma_{0}$ is an arbitrary subboundary, $\partial_{\nu}$ denotes the normal derivative, $0<\theta<T$ and $\ell_{0} \in \mathbb{N}$. In the case of $\ell_{0}=(n+1)^{2} n / 2$, we prove the Lipschitz stability in the inverse problem if we choose $\left(h_{1}, \ldots, h_{\ell_{0}}\right)$ from a set $\mathcal{H} \subset\left\{C_{0}^{\infty}((0, T) \times \omega)\right\}^{\ell_{0}}$ with an arbitrarily fixed subdomain $\omega \subset \Omega$. Moreover we can take $\ell_{0}=(n+3) n / 2$ by making special choices for $h_{\ell}, 1 \leq \ell \leq \ell_{0}$. The proof is based on a Carleman estimate.

Mathematics Subject Classification. 35R30, 35K20.

Received March 7, 2007. Revised December 31, 2007.

Published online July 19, 2008.

\section{INTRODUCTION AND MAIN RESULTS}

In this paper we consider the following parabolic equation:

$$
\begin{gathered}
\partial_{t} y(t, x)-\sum_{i, j=1}^{n} \partial_{j}\left(a_{i j}(x) \partial_{i} y(t, x)\right)=h(t, x), \quad(t, x) \in Q \equiv(0, T) \times \Omega \\
y(t, x)=0, \quad(t, x) \in \Sigma \equiv(0, T) \times \partial \Omega, \quad y(0, \cdot) \in L^{2}(\Omega) .
\end{gathered}
$$

Here $\Omega \subset \mathbb{R}^{n}$ is a bounded domain whose boundary $\partial \Omega$ is sufficiently smooth, and $x=\left(x_{1}, \ldots, x_{n}\right) \in \mathbb{R}^{n}$, $\partial_{t}=\frac{\partial}{\partial t}, \partial_{j}=\frac{\partial}{\partial x_{j}}, \nabla=\left(\partial_{1}, \ldots, \partial_{n}\right), h \in C_{0}^{\infty}((0, T) \times \omega)$, and $\omega$ is an arbitrarily fixed subdomain of $\Omega$. Let $\alpha=\left(\alpha_{1}, \alpha_{2}, \ldots, \alpha_{n}\right)$ be a multi-index with $\alpha_{j} \in \mathbb{N} \cup\{0\}$. We set $\partial_{x}^{\alpha}=\partial_{1}^{\alpha_{1}} \partial_{2}^{\alpha_{2}} \ldots \partial_{n}^{\alpha_{n}}, \quad|\alpha|=\alpha_{1}+\alpha_{2}+\ldots+\alpha_{n}$, and $\nu=\nu(x)=\left(\nu_{1}(x), \ldots, \nu_{n}(x)\right)$ is the external unit normal vector to $\partial \Omega$ at $x$. Let $\partial_{\nu}=\nu \cdot \nabla$.

\footnotetext{
Keywords and phrases. Inverse parabolic problem, Carleman estimate, Lipschitz stability.

1 Department of Mathematical Sciences, The University of Tokyo, Komaba Meguro, Tokyo, 153-8914, Japan. g_h_yuan@hotmail.com; myama@ms.u-tokyo.ac.jp

2 School of Mathematics \& Statistics, Northeast Normal University, Changchun, Jilin, 130024, P. R. China.
} 
Assume that

$$
a_{i j} \in C^{1}(\bar{\Omega}), \quad a_{i j}=a_{j i}, \quad 1 \leq i, j \leq n,
$$

and that the coefficients $\left\{a_{i j}\right\} \equiv\left\{a_{i j}\right\}_{1 \leq i, j \leq n}$ satisfy the uniform ellipticity: there exists a constant $r>0$ such that

$$
\sum_{i, j=1}^{n} a_{i j}(x) \zeta_{i} \zeta_{j} \geq r|\zeta|^{2}, \quad \zeta \in \mathbb{R}^{n}, \quad x \in \bar{\Omega}
$$

For $y(0, \cdot) \in L^{2}(\Omega)$, we can prove (e.g., Pazy [37]) that $y\left(\left\{a_{i j}\right\}, h\right) \in C\left([0, T] ; L^{2}(\Omega)\right) \cap C\left((0, T) ; H^{2}(\Omega) \cap\right.$ $\left.H_{0}^{1}(\Omega)\right) \cap C^{1}\left((0, T) ; L^{2}(\Omega)\right)$ and see also (1.6) below. By $y\left(\left\{a_{i j}\right\}, h\right)(t, x)$ we denote one function satisfying (1.1)(1.2). We note that $y\left(\left\{a_{i j}\right\}, h\right)$ is uniquely determined upon specification of an initial value in $L^{2}(\Omega)$.

We consider the following inverse problem:

Inverse problem. Let $\theta \in(0, T)$ be arbitrarily fixed and $\Gamma_{0} \neq \emptyset$ be an arbitrary relatively open subset of $\partial \Omega$. Select $\ell_{0} \in \mathbb{N}, h_{\ell} \in C_{0}^{\infty}((0, T) \times \omega), 1 \leq \ell \leq \ell_{0}$ suitably and determine $a_{i j}(x), x \in \Omega, 1 \leq i, j \leq n$ by observation data $\left.\partial_{\nu} y\left(\left\{a_{i j}\right\}, h_{\ell}\right)\right|_{(0, T) \times \Gamma_{0}}$ and $y\left(\left\{a_{i j}\right\}, h_{\ell}\right)(\theta, x), x \in \Omega, 1 \leq \ell \leq \ell_{0}$.

We can consider a more general parabolic equation with lower-order terms:

$$
\begin{gathered}
\partial_{t} y(t, x)=\sum_{i, j=1}^{n} \partial_{j}\left(a_{i j}(x) \partial_{i} y(t, x)\right) \\
+\sum_{i=1}^{n} b_{i}(x) \partial_{i} y(t, x)+c(x) y(t, x)+h(t, x), \quad(t, x) \in Q
\end{gathered}
$$

and discuss the determination of $a_{i j}, b_{i}, c, 1 \leq i, j \leq n$ by similar observations. The method is same because a basic estimate (Thm. 2.1) is insensitive to such lower-order terms. However for simplicity, we consider only the determination of the principal part.

In the formulation of the inverse problem, the initial values are also unknown. The non-homogeneous terms $h_{\ell}$, $1 \leq \ell \leq \ell_{0}$, are considered as input sources to system (1.1)-(1.2) and are spatially restricted to a small subdomain $\omega \subset \Omega$. Then we determine $a_{i j}(x), x \in \Omega$ by observation data $\left.\partial_{\nu} y\left(\left\{a_{i j}\right\}, h_{\ell}\right)\right|_{(0, T) \times \Gamma_{0}}$ and $y\left(\left\{a_{i j}\right\}, h_{\ell}\right)(\theta, \cdot)$, $1 \leq \ell \leq \ell_{0}$, which are regarded as outputs.

We shall determine $a_{i j}$ in the neighbourhood of some known set of coefficients $a_{i j}^{(2)}$. We shall denote by $a_{i j}^{(1)}$ the unknown set of coefficients. Solutions associated to $a_{i j}^{(2)}$ will thus be known. The full knowledge of $a_{i j}^{(2)}$ allows for instance to approximately control the solutions to (1.1)-(1.2) associated to $a_{i j}^{(2)}$ with the function $h$ as the control function. In other words, in order to determine $\frac{n(n+1)}{2}$ coefficients $a_{i j}^{(1)}$, we are assumed to be able to operate the heat processes associated to $a_{i j}^{(2)}$ by suitably changing input sources $h_{\ell}$. We note that we need not know initial data in repeating the processes associated to $a_{i j}^{(1)}$, and initial values for the both parabolic equations with $a_{i j}^{(1)}$ and $a_{i j}^{(2)}$, may be arbitrarily changed during the repeated processes. Our main concern is the stability estimate for the inverse problem: Estimate $\sum_{i, j=1}^{n}\left\|a_{i j}^{(1)}-a_{i j}^{(2)}\right\|_{H^{1}(\Omega)}$ by suitable norms of $\partial_{\nu} y\left(\left\{a_{i j}^{(1)}\right\}, h_{\ell}\right)-\partial_{\nu} y\left(\left\{a_{i j}^{(2)}\right\}, h_{\ell}\right)$ and $y\left(\left\{a_{i j}^{(1)}\right\}, h_{\ell}\right)(\theta, \cdot)-y\left(\left\{a_{i j}^{(2)}\right\}, h_{\ell}\right)(\theta, \cdot), 1 \leq \ell \leq \ell_{0}$. The stability is a fundamental mathematical subject in the inverse problem and immediately yields the uniqueness. Stability estimates for inverse problems are not only important from the theoretical viewpoint, but also useful for numerical algorithms. In particular, by Cheng and Yamamoto [10] for example, a stability estimate gives convergence rates of Tikhonov regularized solutions, which are widely used as approximating solutions to the inverse problems. 
Here we assume that initial data are also unknown to be determined. If we can estimate $\sum_{i, j=1}^{n} \| a_{i j}^{(1)}-$ $a_{i j}^{(2)} \|_{H^{1}(\Omega)}$, then we can apply the argument in Yamamoto and Zou [41] (pp. 1187-1188), and we can estimate $y\left(\left\{a_{i j}^{(1)}\right\}, h_{\ell}\right)(0, \cdot)-y\left(\left\{a_{i j}^{(2)}\right\}, h_{\ell}\right)(0, \cdot)$. The argument is concerned with the parabolic equation backward in time. As for the backward heat equation, see the monographs Ames and Straughan [2], Payne [36] and Klibanov [31] as a recent paper. Our main concern is the determination of coefficients and so we will omit the estimation of initial values.

We can consider an inverse problem for a usual initial value/boundary value problem by setting $\theta=0$. In the case where $\theta=0$ and $\Gamma_{0}$ is an arbitrary subboundary of $\Omega$, the corresponding inverse problem is open (e.g., Chap. 9, Sect. 2 in Isakov [25]) even for the inverse problem of determining a single coefficient in a parabolic equation. In the case of $\theta=0$, if $\Gamma_{0} \subset \partial \Omega$ is a sufficiently large portion and unknown coefficients $a_{i j}$ satisfy some additional conditions, then applying an argument in Theorem 4.7 in Klibanov [30], we can prove the stability provided that initial values satisfy some non-degeneracy condition similar to (1.7) below. The additional condition on $a_{i j}^{(2)}$ is described by the pseudo-convexity for the corresponding hyperbolic operator $\partial_{t}^{2}-\sum_{i, j=1}^{n} \partial_{j}\left(a_{i j}^{(2)}(x) \partial_{i}\right)$ (e.g., Chap. VIII, Sect. 5 in Hörmander [18]). Due to the additional conditions on $\Gamma_{0}$ and $a_{i j}^{(2)}$, in the case of $\theta=0$, the available results for the inverse parabolic problem are still incomplete, because the condition on $a_{i j}^{(2)}$ are concerned with the pseudo-convexity for the hyperbolic operator, so that $\Gamma_{0}$ can not be taken arbitrarily. Even if we can prove the stability for the case of $\theta=0$, we have to assume that initial values satisfy some non-degeneracy condition such as (1.7) below stated. From the practical viewpoint, this means that we have to choose such special initial values, which may be difficult in practice. In our case, we need not directly choose values $y\left(\left\{a_{i j}^{(2)}\right\}, h_{\ell}\right)(\theta, \cdot)$, but in order that those values at $t=\theta$ satisfy the requested non-degeneracy condition, we should steer systems by choosing controls which can be limited in any small part of $\Omega$. Therefore we can assert that our formulation is more realizable.

Our inverse problem is related to determination of thermal conductivity of an anisotropic medium by heat conduction process. To the authors' best knowledge, there are no papers on the determination of multiple coefficients in the principal part of a parabolic equation, although we have an available methodology which was initiated by Bukhgeim and Klibanov [8]. The determination of multiple coefficients requires repeat of observations, and the application of the method in [8] needs independent consideration. Moreover, since we aim at the global stability in the whole domain $\Omega$ by means of lateral Cauchy data on an arbitrary small subboundary $\Gamma_{0} \subset \partial \Omega$, we have to establish a relevant Carleman estimate (Thm. 2.1 below).

For statement of our main results, we need to introduce some notations. Let $C^{\ell}(\bar{\Omega}), \ell \in \mathbb{N}$, denote the usual space of functions of $C^{\ell}$-class on $\bar{\Omega}$, and $C^{m-1,1}(\bar{\Omega})$ be the space of all the uniformly Lipschitz continuous functions on $\bar{\Omega}$ with the norm

$$
\|a\|_{C^{m-1,1}(\bar{\Omega})}=\|a\|_{C^{m}(\bar{\Omega})}+\max _{|\alpha|=m} \sup _{x, x^{\prime} \in \Omega, x \neq x^{\prime}} \frac{\left|\partial_{x}^{\alpha} a(x)-\partial_{x}^{\alpha} a\left(x^{\prime}\right)\right|}{\left|x-x^{\prime}\right|} .
$$

For a sequence $\left\{\rho_{\ell}(x)\right\}:=\left\{\rho_{\ell}(x)\right\}_{1 \leq \ell \leq \frac{(n+1)^{2} n}{2}}$ of $C^{2}$-functions and $1 \leq k \leq \frac{n(n+1)}{2}$, we set

$$
\begin{aligned}
D_{i j}^{k} & =D_{i j}^{k}\left(\left\{\rho_{\ell}\right\}\right)(x) \\
& =\operatorname{det}\left(\begin{array}{cccc}
\partial_{i} \partial_{j} \rho_{(k-1)(n+1)+1}(x) & \partial_{1} \rho_{(k-1)(n+1)+1}(x) & \ldots & \partial_{n} \rho_{(k-1)(n+1)+1}(x) \\
\partial_{i} \partial_{j} \rho_{(k-1)(n+1)+2}(x) & \partial_{1} \rho_{(k-1)(n+1)+2}(x) & \ldots & \partial_{n} \rho_{(k-1)(n+1)+2}(x) \\
\vdots & \vdots & \ddots & \vdots \\
\partial_{i} \partial_{j} \rho_{(k-1)(n+1)+n+1}(x) & \partial_{1} \rho_{(k-1)(n+1)+n+1}(x) & \ldots & \partial_{n} \rho_{(k-1)(n+1)+n+1}(x)
\end{array}\right)
\end{aligned}
$$


and

$$
\begin{aligned}
& D\left(\left\{\rho_{\ell}\right\}\right)(x) \\
& =\operatorname{det}\left(\begin{array}{ccccccccc}
D_{11}^{1} & D_{12}^{1} & \ldots & D_{1 n}^{1} & D_{22}^{1} & \ldots & D_{2 n}^{1} & \ldots & D_{n n}^{1} \\
D_{11}^{2} & D_{12}^{2} & \ldots & D_{1 n}^{2} & D_{22}^{2} & \ldots & D_{2 n}^{2} & \ldots & D_{n n}^{2} \\
\vdots & \vdots & \ddots & \vdots & \vdots & \ddots & \vdots & \ddots & \vdots \\
D_{11}^{\frac{1}{2}(n+1) n} & D_{12}^{\frac{1}{2}(n+1) n} & \ldots & D_{1 n}^{\frac{1}{2}(n+1) n} & D_{22}^{\frac{1}{2}(n+1) n} & \ldots & D_{2 n}^{\frac{1}{2}(n+1) n} & \ldots & D_{n n}^{\frac{1}{2}(n+1) n}
\end{array}\right) .
\end{aligned}
$$

Next we introduce an admissible set of unknown coefficients $\left\{a_{i j}\right\}$. We choose $m \in \mathbb{N}$ such that

$$
m>\frac{n}{2}+3
$$

Let us fix constants $M_{0}>0, r>0$ and smooth functions $\eta_{i j}=\eta_{i j}(x), 1 \leq i, j \leq n$ on $\bar{\Omega}$. Let $\omega_{1}=\{x \in \Omega$; $\left.\operatorname{dist}(x, \partial \Omega)<r_{0}\right\}$ with sufficiently small $r_{0}>0$. Then we note that $\partial \omega_{1} \supset \partial \Omega$. Henceforth $[\gamma]$ denotes the greatest integer not exceeding $\gamma \in \mathbb{R}$. We set

$$
\begin{aligned}
\mathcal{U} & =\left\{\left\{a_{i j}\right\} ;\left\|a_{i j}\right\|_{C^{m-1,1}(\bar{\Omega})} \leq M_{0},\right. \\
a_{i j} & \left.=\eta_{i j} \text { in } \omega_{1}, \text { and }(1.3) \text { and (1.4) are satisfied with fixed } r>0\right\} .
\end{aligned}
$$

For $\ell \in \mathbb{N} \cup\{0\}$ and $m_{0} \in\left\{0,1, \ldots, 2\left[\frac{m+1}{2}\right]\right\}$, and $0<\tau_{1}<\tau_{2}<T$, we can prove

$$
\left\|y\left(\left\{a_{i j}\right\}, h\right)\right\|_{C^{\ell}\left(\left[\tau_{1}, \tau_{2}\right] ; H^{m_{0}}(\Omega)\right)} \leq C_{0}\left(\left\|y\left(\left\{a_{i j}\right\}, h\right)(0, \cdot)\right\|_{L^{2}(\Omega)}+\|h\|_{W^{\ell, 1}\left(0, T ; H^{m_{0}}(\omega)\right)}\right) .
$$

Here $C_{0}>0$ depends only $\ell, \tau_{1}, \tau_{2}$ and $\mathcal{U}$, and $\|h\|_{W^{\ell, 1}\left(0, T ; H^{m_{0}}(\omega)\right)}=\sum_{j=0}^{\ell}\left\|\partial_{t}^{j} h\right\|_{L^{1}\left(0, T ; H^{m_{0}}(\omega)\right)}$. The proof relies on semigroup theory (e.g., [37]) and is given in Appendix B.

Remark 1.1. In (1.5), we assume that $a_{i j} \in C^{m-1,1}(\bar{\Omega})$. This can be partly relaxed. However, for the proof, we have to assume that $y\left(\left\{a_{i j}\right\}, h\right)(t, \cdot) \in C^{3}(\bar{\Omega})$ and by semigroup theory we discuss the approximate controllability in $H^{m}(\Omega) \subset C^{3}(\bar{\Omega})$ (by the Sobolev embedding theorem, e.g., Thm. 5.4 in Adams [1]), so that with the Sobolev space we have to relate the regularity of functions in the domain of the operator $A^{\left[\frac{m+1}{2}\right]}$ where the operator $A$ is defined by (1.9) below. For it, we need the regularity in $H^{m}(\Omega)$ for an elliptic equation $\sum_{i, j=1}^{n} \partial_{j}\left(a_{i j}(x) \partial_{i} u(x)\right)=b(x), x \in \Omega\left(e . g\right.$., Chap. 8 in Gilbarg and Trudinger [16]) and $a_{i j} \in C^{m-1,1}(\bar{\Omega})$ is a required regularity condition (e.g., Thm. 8.13, p. 187, in [16]).

Moreover we assume that unknown coefficients $\left\{a_{i j}\right\}$ are given near the boundary $\partial \Omega$, that is, $a_{i j}=\eta_{i j}$ in $\omega_{1}$. This means that we are interested in the determination of coefficients in a compact subset of $\Omega$ away from $\partial \Omega$ with some distance. As is seen from the proof, condition (1.7) below is necessary and the homogeneous Dirichlet boundary condition (1.2) implies that (1.7) does not hold on $\partial \Omega$, because there exist zero column vectors of $D\left(\left\{y\left(\left\{a_{i j}^{(2)}\right\}, h_{\ell}\right)\right\}\right)$. This technically motivates that we discuss the determination of $\left\{a_{i j}^{(1)}\right\}$ on $\overline{\Omega \backslash \omega_{1}}$, and that we assume $a_{i j}^{(1)}=\eta_{i j}$ in $\omega_{1}$. We further note that since we consider solutions in a time interval away from $t=0$, we can improve the regularity in $t \in\left(\tau_{1}, \tau_{2}\right)$ as we wish (see the proof of (1.6) in Appendix B), while the $x$-regularity in $H^{m}(\Omega)$ with $m>\frac{n}{2}+3$, is necessary for our argument.

Henceforth, for an arbitrarily fixed $M>0$, we assume that

$$
\left\|y\left(\left\{a_{i j}^{(j)}\right\}, h\right)(0, \cdot)\right\|_{L^{2}(\Omega)} \leq M, \quad j=1,2,
$$

which means that the unknown initial values are bounded with an a priori bound $M>0$.

Now we are ready to state our main results. 
Theorem 1.1. Let $0<\tau_{1}<\theta<\tau_{2}<T, \Gamma_{0} \neq \emptyset$ be an arbitrary relatively open subset of $\partial \Omega$, and let $\left\{a_{i j}^{(2)}\right\} \in \mathcal{U}$ be arbitrarily fixed. We assume that $h_{\ell} \in C_{0}^{\infty}((0, T) \times \omega), 1 \leq \ell \leq \frac{(n+1)^{2} n}{2}$, satisfy

$$
D\left(\left\{y\left(\left\{a_{i j}^{(2)}\right\}, h_{\ell}\right)\right\}\right)(\theta, x) \neq 0
$$

for $x \in \overline{\Omega \backslash \omega_{1}}$. Then there exists a constant $C_{1}=C_{1}\left(\mathcal{U}, M,\left\{h_{\ell}\right\}\right)>0$ such that

$$
\begin{aligned}
& \sum_{i, j=1}^{n}\left\|a_{i j}^{(1)}-a_{i j}^{(2)}\right\|_{H^{1}(\Omega)} \leq C_{1} \sum_{\ell=1}^{\frac{(n+1)^{2} n}{2}}\left\|\partial_{\nu} y\left(\left\{a_{i j}^{(1)}\right\}, h_{\ell}\right)-\partial_{\nu} y\left(\left\{a_{i j}^{(2)}\right\}, h_{\ell}\right)\right\|_{H^{2}\left(\tau_{1}, \tau_{2} ; L^{2}\left(\Gamma_{0}\right)\right)} \\
& +C_{1} \sum_{\ell=1}^{\frac{(n+1)^{2} n}{2}}\left\|y\left(\left\{a_{i j}^{(1)}\right\}, h_{\ell}\right)(\theta, \cdot)-y\left(\left\{a_{i j}^{(2)}\right\}, h_{\ell}\right)(\theta, \cdot)\right\|_{H^{3}(\Omega)}
\end{aligned}
$$

for all $\left\{a_{i j}^{(1)}\right\} \in \mathcal{U}$.

In order to estimate $\left\{a_{i j}^{(1)}\right\}$ around a given $\left\{a_{i j}^{(2)}\right\}$, we have to choose $h_{\ell}, 1 \leq \ell \leq \frac{(n+1)^{2} n}{2}$ whose supports are restricted to a small set $(0, T) \times \omega$, so that the systems are steered to satisfy (1.7) on $\overline{\Omega \backslash \omega_{1}}$ at the time $\theta$. The choice is related to approximate controllability of parabolic equations (e.g., [39]).

Henceforth we define an operator $A$ in $L^{2}(\Omega)$ by

$$
\left\{\begin{array}{l}
(A y)(x)=-\sum_{i, j=1}^{n} \partial_{j}\left(a_{i j}(x) \partial_{i} y(x)\right), \quad x \in \Omega, \\
\mathcal{D}(A)=H^{2}(\Omega) \cap H_{0}^{1}(\Omega),
\end{array}\right.
$$

where $\mathcal{D}(A)$ denotes the domain of the operator $A$, and let $y\left(\left\{a_{i j}\right\}, h, \mu\right)$ denote the solution to (1.1) and (1.2) with $y(0, x)=\mu(x), x \in \Omega$.

Then we can prove:

Proposition 1.1. Let $m_{1}=\left[\frac{m+1}{2}\right]$, that is, $m_{1}=\frac{m}{2}$ if $m$ is even and $m_{1}=\frac{m+1}{2}$ if $m$ is odd. Let $\left\{a_{i j}\right\} \in \mathcal{U}$. For each $\theta>0$ and $\mu \in L^{2}(\Omega)$, the set

$$
\left\{y\left(\left\{a_{i j}\right\}, h, \mu\right)(\theta, \cdot) ; h \in C_{0}^{\infty}((0, T) \times \omega)\right\}
$$

is dense in $\mathcal{D}\left(A^{m_{1}}\right)=\left\{y \in H^{2 m_{1}}(\Omega) ;\left.A^{j} y\right|_{\partial \Omega}=0,0 \leq j \leq m_{1}-1\right\}$.

By Proposition 1.1, we can prove the existence of $h_{\ell} \in C_{0}^{\infty}((0, T) \times \omega), 1 \leq \ell \leq \frac{(n+1)^{2} n}{2}$ such that (1.7) holds on $\overline{\Omega \backslash \omega_{1}}$, which guarantees the Lipschitz stability in determining $\left\{a_{i j}^{(1)}\right\}$. In fact, we arbitrarily choose $\left\{\rho_{\ell}\right\}_{1 \leq \ell \leq \frac{(n+1)^{2} n}{2}} \subset C_{0}^{\infty}(\Omega)$ such that $\operatorname{det} D\left(\left\{\rho_{\ell}\right\}\right)(x) \neq 0$ for $x \in \overline{\Omega \backslash \omega_{1}}$. We note that $\rho_{\ell} \in \mathcal{D}\left(A^{m_{1}}\right)$. In terms of Proposition 1.1, for sufficiently small $\varepsilon>0$, we can fix $h_{\ell} \in C_{0}^{\infty}((0, T) \times \omega), 1 \leq \ell \leq \frac{(n+1)^{2} n}{2}$ satisfying

$$
\left\|y\left(\left\{a_{i j}^{(2)}\right\}, h_{\ell}\right)(\theta, \cdot)-\rho_{\ell}\right\|_{H^{2 m_{1}(\Omega)}}<\varepsilon, \quad 1 \leq \ell \leq \frac{(n+1)^{2} n}{2} .
$$

By the Sobolev embedding theorem, we have

$$
\left\|y\left(\left\{a_{i j}^{(2)}\right\}, h_{\ell}\right)(\theta, \cdot)-\rho_{\ell}\right\|_{C^{2}(\bar{\Omega})}<C \varepsilon, \quad 1 \leq \ell \leq \frac{(n+1)^{2} n}{2} .
$$


By $\operatorname{det} D\left(\left\{\rho_{\ell}\right\}\right)(x) \neq 0$ for $x \in \overline{\Omega \backslash \omega_{1}}$, we obtain (1.7) on $\overline{\Omega \backslash \omega_{1}}$ for sufficiently small $\varepsilon>0$. The control functions $h_{\ell}$ can be constructed in practice by means of the methods in Fabre et al. [14], Glowinski and Lions [17].

Now we discuss the set of such $h_{\ell}, 1 \leq \ell \leq \frac{(n+1)^{2} n}{2}$. For simplicity, for the system with known $a_{i j}^{(2)}$, we assume the zero initial value. That is, we let $y\left(\left\{a_{i j}^{(2)}\right\}, h, 0\right)$ be the unique solution to (1.1) and (1.2) with $y(x, 0)=0$, $x \in \Omega$. We set $\ell_{0}=\frac{(n+1)^{2} n}{2}$ and

$$
\mathcal{H}=\left\{\left(h_{1}, \ldots, h_{\ell_{0}}\right) \in\left\{C_{0}^{\infty}((0, T) \times \omega)\right\}^{\ell_{0}} ; D\left(\left\{y\left(\left\{a_{i j}^{(2)}\right\}, h_{\ell}, 0\right)\right\}\right)(\theta, x) \neq 0 \quad \text { for } x \in \overline{\Omega \backslash \omega_{1}} .\right\}
$$

From elliptic regularity (e.g., Thm. 8.13 in [16]) and semigroup theory (e.g., [37]), we can prove that there exists a constant $C_{2}>0$ such that

$$
\left\|y\left(\left\{a_{i j}\right\}, h, 0\right)\right\|_{C\left([0, T] ; C^{2}(\bar{\Omega})\right)} \leq C_{2}\|h\|_{L^{1}\left(0, T ; H^{m}(\Omega)\right)},
$$

where the constant $C_{2}$ can be taken uniformly for $\left\{a_{i j}\right\} \in \mathcal{U}$. See Appendix B for the proof.

Therefore we can prove that for $\left(h_{1}, \ldots, h_{\ell_{0}}\right) \in \mathcal{H}$, there exists $\varepsilon=\varepsilon\left(h_{1}, \ldots, h_{\ell_{0}}\right)>0$ such that if $\left(\widetilde{h}_{1}, \ldots, \widetilde{h}_{\ell_{0}}\right) \in$ $\left\{C_{0}^{\infty}((0, T) \times \omega)\right\}^{\ell_{0}}$ and $\max _{1 \leq \ell \leq \ell_{0}}\left\|h_{\ell}-\widetilde{h}_{\ell}\right\|_{L^{1}\left(0, T ; H^{m}(\Omega)\right)}<\varepsilon$, then $\left(\widetilde{h}_{1}, \ldots, \widetilde{h}_{\ell_{0}}\right) \in \mathcal{H}$ by the definition of $D\left(\left\{y\left(\left\{a_{i j}^{(2)}\right\}, h_{\ell}, 0\right)\right\}\right)(\theta, x)$. This means the stability of input sources $\left(h_{1}, \ldots, h_{\ell_{0}}\right)$ realizing the Lipschitz stability.

Since $C_{0}^{\infty}((0, T) \times \omega)$ is dense in $C_{0}^{\ell}((0, T) \times \omega)$ with $\ell \in \mathbb{N}$, we can take $C_{0}^{\ell}((0, T) \times \omega)$ as a class of interior input sources, using parabolic regularity properties (e.g., [37]).

Furthermore we can prove an even better result with smaller $\ell_{0}$ in Theorem 1.1. That is, with arbitrary initial values for system (1.1) associated to the set of coefficients $a_{i j}^{(2)}$, we can choose $h_{\ell}, 1 \leq \ell \leq \frac{(n+3) n}{2}$ to establish the Lipschitz stability around $a_{i j}^{(2)}$ by means of $\frac{(n+3) n}{2}$ data. The choice of such $h_{\ell}$ is different from Theorem 1.1, but Proposition 1.1 guarantees that such a choice is possible.

Theorem 1.2. Let $0<\tau_{1}<\theta<\tau_{2}<T, \Gamma_{0} \neq \emptyset$ be an arbitrary relatively open subset of $\partial \Omega$ and let us fix $\left\{a_{i j}^{(2)}\right\} \in \mathcal{U}$. Then we can choose suitable $h_{\ell} \in C_{0}^{\infty}((0, T) \times \omega), 1 \leq \ell \leq \frac{n(n+3)}{2}$ such that there exists a constant $C_{2}=C_{2}\left(\mathcal{U}, M,\left\{h_{\ell}\right\}\right)>0$ such that

$$
\begin{aligned}
& \sum_{i, j=1}^{n}\left\|a_{i j}^{(1)}-a_{i j}^{(2)}\right\|_{H^{1}(\Omega)} \leq C_{2} \sum_{\ell=1}^{\frac{n(n+3)}{2}}\left\|\partial_{\nu} y\left(\left\{a_{i j}^{(1)}\right\}, h_{\ell}\right)-\partial_{\nu} y\left(\left\{a_{i j}^{(2)}\right\}, h_{\ell}\right)\right\|_{H^{2}\left(\tau_{1}, \tau_{2} ; L^{2}\left(\Gamma_{0}\right)\right)} \\
& +C_{2} \sum_{\ell=1}^{\frac{n(n+3)}{2}}\left\|y\left(\left\{a_{i j}^{(1)}\right\}, h_{\ell}\right)(\theta, \cdot)-y\left(\left\{a_{i j}^{(2)}\right\}, h_{\ell}\right)(\theta, \cdot)\right\|_{H^{3}(\Omega)}
\end{aligned}
$$

for all $\left\{a_{i j}^{(1)}\right\} \in \mathcal{U}$.

Since the number of the unknown coefficients is $\frac{n(n+1)}{2}$, it is natural to expect that suitable $\frac{n(n+1)}{2}$-times observations can yield the Lipschitz stability, and even the result in Theorem 1.2 holds with overdetermining observations (i.e., $\frac{n(n+3)}{2}$-times observations). We do not presently know whether we can reduce the number of observations to $\frac{n(n+1)}{2}$. In particular, for the case $a_{i j}(x)=\left\{\begin{array}{ll}a(x), & i=j, \\ 0, & i \neq j,\end{array}\right.$ we can prove that a single observation by a suitable single input $h_{1}$ yields the Lipschitz stability. The proof is done similarly to Imanuvilov and Yamamoto [23] where an inverse problem for an acoustic equation $\partial_{t}^{2} u=\operatorname{div}(a(x) \nabla u)$ is discussed. In (1.11), we can replace $\left\|a_{i j}^{(1)}-a_{i j}^{(2)}\right\|_{H^{1}(\Omega)}$ by a weaker norm $\left\|a_{i j}^{(1)}-a_{i j}^{(2)}\right\|_{L^{2}(\Omega)}$ and can adopt the corresponding weaker norms of observation data. As is stated as Theorem 2.1, our basic tool is an $L^{2}$-weighted estimate called a Carleman estimate where the right-hand side is estimated by an $L^{2}$-weighted norm. We can prove a similar Carleman estimate where the right-hand side is estimated in an $H^{-1}$-weighted space (Imanuvilov and 
Yamamoto [22,24]). Then $\left\|a_{i j}^{(1)}-a_{i j}^{(2)}\right\|_{L^{2}(\Omega)}$ can be estimated by such an $H^{-1}$-Carleman estimate by a method similar to [23]. However we do not still know whether we can reduce the number of observations in the case of $\left\|a_{i j}^{(1)}-a_{i j}^{(2)}\right\|_{L^{2}(\Omega)}$

As for inverse problems of determining coefficients in parabolic equations, we refer to Danilaev [11], Elayyan and Isakov [12], Imanuvilov and Yamamoto [20,22], Isakov [25], Isakov and Kindermann [26], Ivanchov [27], Klibanov [30], Klibanov and Timonov [32], Yamamoto and Zou [41]. In particular, in [12,26,30], determination problems for principal parts are discussed. In those existing papers, the determination of a single coefficient is discussed, while we here consider an inverse problem of determining multiple coefficients of the principal part by a finite set of observations.

Our formulation is with a finite number of observations and this kind of inverse problems was firstly solved by Bukhgeim and Klibanov [8], whose methodology is based on Carleman estimates. For similar inverse problems for other equations, we refer to Baudouin and Puel [3], Bellassoued [4], Bellassoued and Yamamoto [5], Bukhgeim [7], Imanuvilov and Yamamoto [21,23], Isakov [25], Khal̆darov [28], Klibanov [29,30], Klibanov and Timonov [32], Klibanov and Yamamoto [33], Yamamoto [40].

For proving Theorems 1.1 and 1.2, we establish a Carleman estimate (Thm. 2.1) for functions with noncompact support, and we apply a modification of arguments in [8,23].

This paper is composed of four sections and three appendices. In Section 2 we present Carleman estimates and the proof is given in Appendix A. In Section 3, we prove Theorems 1.1 and 1.2. In Section 4, we prove Proposition 1.1. In Appendix B, we prove estimates (1.6) and (1.10). Appendix C is devoted to the proof of the existence of a suitable weight function for our Carleman estimate.

\section{Carleman estimates}

In this section we will prove Carleman estimates for the parabolic equation. The results in this section may have independent interests.

Lemma 2.1. Let $\Gamma_{0} \neq \emptyset \subset \partial \Omega$ be an arbitrary relatively open subset. Then there exists a function $d \in C^{2}(\bar{\Omega})$ such that

$$
d(x)>0 \quad \text { for } x \in \Omega, \quad|\nabla d(x)|>0 \quad \text { for } x \in \bar{\Omega}
$$

and

$$
\sum_{i, j=1}^{n} a_{i j}(x) \partial_{i} d(x) \nu_{j}(x) \leq 0, \quad x \in \partial \Omega \backslash \Gamma_{0}
$$

for all $a_{i j} \in C^{1}(\bar{\Omega}), a_{i j}=a_{j i}, 1 \leq i, j \leq n$ satisfying (1.4).

Lemma 2.1 can be derived directly from Lemma 1.2 in [19] where $d(x)>0$ is not stated, and for convenience we prove this lemma in Appendix C.

Example 2.1. Let us consider a special case where $a_{i j}=0$ if $i \neq j$ and $a_{i i}=1$ and

$$
\Omega=\left\{x \in \mathbb{R}^{n} ;|x|<R\right\}, \quad \Gamma_{0}=\left\{x \in \partial \Omega ;\left(x-x_{0}, \nu(x)\right) \geq 0\right\}
$$

with an arbitrarily fixed $x_{0} \in \mathbb{R}^{n} \backslash \bar{\Omega}$. Here $(\cdot, \cdot)$ denotes the scalar product in $\mathbb{R}^{n}$. Then we can take $d(x)=$ $\left|x-x_{0}\right|^{2}$.

We present Carleman estimates for an operator $L$ :

$$
(L y)(t, x)=\partial_{t} y(t, x)-\sum_{i, j=1}^{n} \partial_{j}\left(a_{i j}(x) \partial_{i} y(t, x)\right)
$$


Theorem 2.1. Assume that (1.4) holds and that $a_{i j} \in C^{1}(\bar{\Omega}), a_{i j}=a_{j i}, 1 \leq i, j \leq n$. Let $d \in C^{2}(\bar{\Omega})$ be a function satisfying (2.1) and (2.2), and let $0 \leq \tau_{1}<\theta<\tau_{2}$ be fixed.

(1) Let $\varphi(t, x)=\mathrm{e}^{\lambda\left(d(x)-\beta|t-\theta|^{2}\right)}$, where $\beta>0$ is a constant. Then there exists a number $\lambda_{0}>0$ such that for an arbitrary $\lambda \geq \lambda_{0}$, we can choose a constant $s_{0}(\lambda) \geq 0$ satisfying: there exists a constant $C_{1}=C_{1}\left(s_{0}, \lambda\right)>0$ such that

$$
\begin{aligned}
& \int_{\left(\tau_{1}, \tau_{2}\right) \times \Omega}\left\{\frac{1}{s}\left(\left|\partial_{t} v\right|^{2}+\sum_{i, j=1}^{n}\left|\partial_{i} \partial_{j} v\right|^{2}\right)+s|\nabla v|^{2}+s^{3}|v|^{2}\right\} \mathrm{e}^{2 s \varphi} \mathrm{d} x \mathrm{~d} t \\
& \leq C_{1} \int_{\left(\tau_{1}, \tau_{2}\right) \times \Omega}|L v|^{2} \mathrm{e}^{2 s \varphi} \mathrm{d} x \mathrm{~d} t+C_{1} s \int_{\tau_{1}}^{\tau_{2}} \int_{\Gamma_{0}}\left|\partial_{\nu} v\right|^{2} \mathrm{e}^{2 s \varphi} \mathrm{d} \Sigma
\end{aligned}
$$

for all $s>s_{0}$ and all $v$ satisfying

$$
\left\{\begin{array}{l}
L v \in L^{2}\left(\left(\tau_{1}, \tau_{2}\right) \times \Omega\right), \quad v \in L^{2}\left(\tau_{1}, \tau_{2} ; H^{2}(\Omega) \cap H_{0}^{1}(\Omega)\right), \\
v\left(\tau_{1}, \cdot\right)=v\left(\tau_{2}, \cdot\right)=0 .
\end{array}\right.
$$

Moreover the constants $s_{0}$ and $C_{1}$ continuously depend on $\lambda$ and $\sum_{i, j=1}^{n}\left\|a_{i j}\right\|_{C^{1}(\bar{\Omega})}, \tau_{1}, \tau_{2}, \Omega, r$, while $\lambda_{0}$ continuously depends on $\sum_{i, j=1}^{n}\left\|a_{i j}\right\|_{C^{1}(\bar{\Omega})}, \tau_{1}, \tau_{2}, \Omega, r$.

(2) Let $\varphi(t, x)=\mathrm{e}^{\lambda\left(d(x)-\beta|t-\theta|^{2}+M_{1}\right)}$, where $M_{1}>\sup _{t \in\left(\tau_{1}, \tau_{2}\right)} \beta(t-\theta)^{2}$. Then there exist positive constants $\lambda_{0}, s_{0}$ and $C_{2}=C_{2}\left(\lambda_{0}, s_{0}\right)$ such that

$$
\begin{aligned}
& \int_{\left(\tau_{1}, \tau_{2}\right) \times \Omega}\left\{\frac{1}{s \varphi}\left(\left|\partial_{t} v\right|^{2}+\sum_{i, j=1}^{n}\left|\partial_{i} \partial_{j} v\right|^{2}\right)+s \lambda^{2} \varphi|\nabla v|^{2}+s^{3} \lambda^{4} \varphi^{3}|v|^{2}\right\} \mathrm{e}^{2 s \varphi} \mathrm{d} x \mathrm{~d} t \\
& \leq C_{2} \int_{\left(\tau_{1}, \tau_{2}\right) \times \Omega}|L v|^{2} \mathrm{e}^{2 s \varphi} \mathrm{d} x \mathrm{~d} t+C_{2} s \lambda \int_{\tau_{1}}^{\tau_{2}} \int_{\Gamma_{0}} \varphi\left|\partial_{\nu} v\right|^{2} \mathrm{e}^{2 s \varphi} \mathrm{d} \Sigma
\end{aligned}
$$

for all $s>s_{0}, \lambda>\lambda_{0}$ and all $v$ satisfying (2.5). The constants $\lambda_{0}, s_{0}$ and $C_{2}$ continuously depend on $\sum_{i, j=1}^{n}\left\|a_{i j}\right\|_{C^{1}(\bar{\Omega})}, \tau_{1}, \tau_{2}, \Omega, r$.

We prove the theorem in Appendix A.

As for Carleman estimates with regular weight function $\varphi(t, x)$, see Eller and Isakov [13], Hörmander [18], Isakov [25], Khă̌darov [28], Klibanov and Timonov [32], Lavrent'ev, Romanov and Shishat.skiü [34]. With these Carleman estimates for parabolic equations, we often have to change independent variables to address the case of an arbitrary subboundary $\Gamma_{0}$ of the boundary $\partial \Omega$. As a result, it becomes much more complicated to obtain a Lipschitz stability estimate over $\Omega \backslash \omega_{1}$, for the coefficients which one tries to identify. As for Carleman estimates for parabolic equations with singular weight function $\varphi(t, x)$, we can refer to Fursikov and Imanuvilov [15], Imanuvilov [19], Imanuvilov and Yamamoto [22,24], and such Carleman estimates hold for a function $v$ not satisfying $v\left(\tau_{1}, \cdot\right)=v\left(\tau_{2}, \cdot\right)=0$.

Inequality (2.6) is a Carleman estimate for functions with non-compact support, and estimates the left-hand side with the weighted $L^{2}$-norms of $L v$ in $\left(\tau_{1}, \tau_{2}\right) \times \Omega$ and $\partial_{\nu} v$ on $\left(\tau_{1}, \tau_{2}\right) \times \Gamma_{0}$. Once we can prove a Carleman estimate for functions with compact support, we can immediately estimate functions with non-compact support by means of a cut-off function, but the norm of the boundary value is stronger that the weighted $L^{2}$-norm, and any Carleman estimates for functions with compact support, does not give a better estimate for our inverse problem.

Thanks to two large parameters $\lambda, s$ and the form of the weight function, Carleman estimate (2.6) can be applied to inverse problems for a coupling system of parabolic and hyperbolic equations and thermoelastic plate 
equations in case (2.3) for example. For such applicability, we will prove Carleman estimates with regular $\psi(t, x)$ as Theorem 2.1.

As for Carleman estimates with two large parameters for functions with compact support, we can refer to [13].

\section{Proofs of Theorems 1.1 And 1.2}

Proof of Theorem 1.1. By $0<\tau_{1}<\tau_{2}<T$, we choose and fix $\tau_{3}, \tau_{4}>0$ such that

$$
0<\tau_{3}<\tau_{1}<\tau_{2}<\tau_{4}<T \text {. }
$$

It is sufficient to prove (1.8) with the norm in $H^{2}\left(\tau_{3}, \tau_{4} ; L^{2}\left(\Gamma_{0}\right)\right)$ of the first term on the righ-hand side. Let $d \in C^{2}(\bar{\Omega})$ satisfy (2.1) and (2.2). We choose $\beta>0$ such that $\sup _{x \in \Omega} d(x)<\beta \min \left\{\left|\tau_{1}-\theta\right|^{2},\left|\tau_{2}-\theta\right|^{2}\right\}$. We set

$$
\varphi(t, x)=\exp \left\{\lambda\left(d(x)-\beta|t-\theta|^{2}\right)\right\} .
$$

Let $d_{0}=\inf _{x \in \Omega} \exp \{\lambda d(x)\} \geq 1$. Then, by the choice of $\beta>0$, we have

$$
\varphi(\theta, x) \geq d_{0}, \quad \varphi\left(\tau_{1}, x\right), \varphi\left(\tau_{2}, x\right)<1 \leq d_{0}, \quad x \in \bar{\Omega} .
$$

Thus for a sufficiently small $\varepsilon>0$, we can choose a small $\delta=\delta(\varepsilon)>0$ such that $\tau_{1}<\tau_{1}+2 \delta<\theta-\delta<\theta+\delta<$ $\tau_{2}-2 \delta<\tau_{2}$,

$$
\varphi(t, x) \geq d_{0}-\varepsilon, \quad(t, x) \in[\theta-\delta, \theta+\delta] \times \bar{\Omega}
$$

and

$$
\varphi(t, x) \leq d_{0}-2 \varepsilon, \quad(t, x) \in\left(\left[\tau_{1}, \tau_{1}+2 \delta\right] \cup\left[\tau_{2}-2 \delta, \tau_{2}\right]\right) \times \bar{\Omega} .
$$

We introduce a cut-off function $\chi$ satisfying $0 \leq \chi \leq 1, \chi \in C_{0}^{\infty}(0, T)$ and

$$
\chi(t)= \begin{cases}0, & t \in\left[\tau_{1}, \tau_{1}+\delta\right] \cup\left[\tau_{2}-\delta, \tau_{2}\right], \\ 1, & t \in\left[\tau_{1}+2 \delta, \tau_{2}-2 \delta\right] .\end{cases}
$$

Let us set

$$
\begin{gathered}
f_{i j}(x)=a_{i j}^{(1)}(x)-a_{i j}^{(2)}(x), \quad R_{\ell}(t, x)=y\left(\left\{a_{i j}^{(2)}\right\}, h_{\ell}\right)(t, x), \\
\left(L^{(1)} y\right)(t, x) \equiv \partial_{t} y-\sum_{i, j=1}^{n} \partial_{j}\left(a_{i j}^{(1)}(x) \partial_{i} y\right) .
\end{gathered}
$$

By (1.1) and (1.2), we can see that the differences

$$
\widetilde{y}_{\ell}(t, x)=y\left(\left\{a_{i j}^{(1)}\right\}, h_{\ell}\right)(t, x)-y\left(\left\{a_{i j}^{(2)}\right\}, h_{\ell}\right)(t, x)
$$

satisfy

$$
\begin{gathered}
L^{(1)} \widetilde{y}_{\ell}(t, x)=\sum_{i, j=1}^{n} \partial_{j}\left(f_{i j}(x) \partial_{i} R_{\ell}(t, x)\right), \quad(t, x) \in(0, T) \times \Omega, \\
\widetilde{y}_{\ell}(t, x)=0, \quad(t, x) \in(0, T) \times \partial \Omega, \quad 1 \leq \ell \leq \frac{(n+1)^{2} n}{2} .
\end{gathered}
$$

We set

$$
z_{\ell}(t, x)=\partial_{t} \widetilde{y}_{\ell}(t, x), \quad \Phi=\sup _{(t, x) \in\left(\tau_{1}, \tau_{2}\right) \times \Omega} \varphi(t, x)
$$


and

$$
\begin{gathered}
U=\left(\sum _ { \ell = 1 } ^ { \frac { ( n + 1 ) ^ { 2 } n } { 2 } } \left(\left\|z_{\ell}\right\|_{L^{2}\left(\tau_{1}, \tau_{2} ; L^{2}(\Omega)\right)}^{2}+\left\|\nabla z_{\ell}\right\|_{L^{2}\left(\tau_{1}, \tau_{2} ; L^{2}(\Omega)\right)}^{2}\right.\right. \\
\left.\left.+\left\|\partial_{t} z_{\ell}\right\|_{L^{2}\left(\tau_{1}, \tau_{2} ; L^{2}(\Omega)\right)}^{2}+\left\|\nabla \partial_{t} z_{\ell}\right\|_{L^{2}\left(\tau_{1}, \tau_{2} ; L^{2}(\Omega)\right)}^{2}\right)\right)^{\frac{1}{2}} \\
V=\left(\sum_{\ell=1}^{\frac{(n+1)^{2} n}{2}}\left\|\partial_{\nu} \widetilde{y}_{\ell}\right\|_{H^{2}\left(\tau_{1}, \tau_{2} ; L^{2}\left(\Gamma_{0}\right)\right)}^{2}\right)^{\frac{1}{2}} .
\end{gathered}
$$

Then by (3.1), (3.3) and (3.5), we have

$$
L^{(1)}\left(z_{\ell} \chi\right)=\sum_{i, j=1}^{n} \partial_{j}\left(\chi f_{i j}(x) \partial_{i} \partial_{t} R_{\ell}(t, x)\right)+z_{\ell} \partial_{t} \chi
$$

and

$$
L^{(1)}\left(\chi \partial_{t} z_{\ell}\right)=\sum_{i, j=1}^{n} \partial_{j}\left(\chi f_{i j}(x) \partial_{i} \partial_{t}^{2} R_{\ell}(t, x)\right)+\left(\partial_{t} \chi\right) \partial_{t} z_{\ell}
$$

We set

$$
Q_{1}=\left(\tau_{1}, \tau_{2}\right) \times \Omega .
$$

Let $1 \leq \ell \leq \frac{(n+1)^{2} n}{2}$. By (1.6), we see that $y\left(\left\{a_{i j}^{(k)}\right\}, h_{\ell}\right) \in C^{3}\left(\left[\tau_{1}, \tau_{2}\right] ; H^{m}(\Omega)\right), k=1,2$, so that the right-hand sides of (3.6) and (3.7) are in $L^{2}\left(Q_{1}\right)$. Moreover from (3.4) it follows that $\chi \partial_{t} z_{\ell}, \chi z_{\ell} \in C\left(\left[\tau_{1}, \tau_{2}\right] ; H^{2}(\Omega) \cap H_{0}^{1}(\Omega)\right)$. Furthermore, by $(3.1)$, we have $\left(\chi \partial_{t} z_{\ell}\right)\left(\tau_{1}, \cdot\right)=\left(\chi z_{\ell}\right)\left(\tau_{1}, \cdot\right)=\left(\chi \partial_{t} z_{\ell}\right)\left(\tau_{2}, \cdot\right)=\left(\chi z_{\ell}\right)\left(\tau_{2}, \cdot\right)=0$.

Henceforth $C_{j}$ denote generic constants which are dependent on $\Omega, T, \tau_{1}, \tau_{2}, r, \lambda, M, \mathcal{U},\left\{h_{\ell}\right\}$, but independent of $s$. We can apply Theorem 2.1.(1) to (3.6) and (3.7) in $Q_{1}$. Then

$$
\begin{aligned}
& \int_{Q_{1}}\left\{s\left|\nabla\left(\chi z_{\ell}\right)\right|^{2}+s^{3}\left|\chi z_{\ell}\right|^{2}\right\} \mathrm{e}^{2 s \varphi} \mathrm{d} x \mathrm{~d} t \leq C_{1} \sum_{i, j=1}^{n} \sum_{|\alpha| \leq 1} \int_{Q_{1}} \chi^{2}\left|\partial_{x}^{\alpha} f_{i j}\right|^{2} \mathrm{e}^{2 s \varphi} \mathrm{d} x \mathrm{~d} t \\
& +C_{1} U^{2} \mathrm{e}^{2 s\left(d_{0}-2 \varepsilon\right)}+C_{1} s \int_{\tau_{1}}^{\tau_{2}} \int_{\Gamma_{0}}\left|\partial_{\nu}\left(\chi z_{\ell}\right)\right|^{2} \mathrm{e}^{2 s \varphi} \mathrm{d} \Sigma, \quad s \geq s_{0}
\end{aligned}
$$

and

$$
\begin{aligned}
& \int_{Q_{1}}\left\{s\left|\nabla\left(\chi \partial_{t} z_{\ell}\right)\right|^{2}+s^{3}\left|\chi \partial_{t} z_{\ell}\right|^{2}\right] \mathrm{e}^{2 s \varphi} \mathrm{d} x \mathrm{~d} t \leq C_{1} \sum_{i, j=1}^{n} \sum_{|\alpha| \leq 1} \int_{Q_{1}} \chi^{2}\left|\partial_{x}^{\alpha} f_{i j}(x)\right|^{2} \mathrm{e}^{2 s \varphi} \mathrm{d} x \mathrm{~d} t \\
& +C_{1} U^{2} \mathrm{e}^{2 s\left(d_{0}-2 \varepsilon\right)}+C_{1} s \int_{\tau_{1}}^{\tau_{2}} \int_{\Gamma_{0}}\left|\partial_{\nu}\left(\chi \partial_{t} z_{\ell}\right)\right|^{2} \mathrm{e}^{2 s \varphi} \mathrm{d} \Sigma, \quad s \geq s_{0}
\end{aligned}
$$


Here we have used that $\partial_{t} \chi \neq 0$ only if $\varphi(t, x) \leq d_{0}-2 \varepsilon$. On the other hand, we have

$$
\begin{aligned}
& \int_{\Omega}\left|\partial_{t} \widetilde{y}_{\ell}(\theta, x)\right|^{2} \mathrm{e}^{2 s \varphi(\theta, x)} \mathrm{d} x=\int_{\Omega}\left|\chi(\theta) \partial_{t} \widetilde{y}_{\ell}(\theta, x)\right|^{2} \mathrm{e}^{2 s \varphi(\theta, x)} \mathrm{d} x \\
& =\int_{\tau_{1}}^{\theta} \partial_{t}\left(\int_{\Omega}\left|\chi(t) \partial_{t} \widetilde{y}_{\ell}(t, x)\right|^{2} \mathrm{e}^{2 s \varphi(t, x)} \mathrm{d} x\right) \mathrm{d} t \\
& \leq \int_{Q_{1}} 2\left(\left|\partial_{t}^{2} \widetilde{y}_{\ell}\right|\left|\partial_{t} \widetilde{y}_{\ell}\right| \chi^{2}+s\left|\partial_{t} \varphi\right|\left|\chi \partial_{t} \widetilde{y}_{\ell}\right|^{2}\right) \mathrm{e}^{2 s \varphi} \mathrm{d} x \mathrm{~d} t+\int_{Q_{1}} 2\left|\partial_{t} \widetilde{y}_{\ell}\right|^{2} \chi\left|\partial_{t} \chi\right| \mathrm{e}^{2 s \varphi} \mathrm{d} x \mathrm{~d} t \\
& \leq C_{2} \int_{Q_{1}}\left|\chi \partial_{t} z_{\ell}\right|^{2} \mathrm{e}^{2 s \varphi(t, x)} \mathrm{d} x \mathrm{~d} t+C_{2}(s+1) \int_{Q_{1}}\left|\chi z_{\ell}\right|^{2} \mathrm{e}^{2 s \varphi(t, x)} \mathrm{d} x \mathrm{~d} t+C_{2} U^{2} \mathrm{e}^{2 s\left(d_{0}-2 \varepsilon\right)} .
\end{aligned}
$$

By (3.8)-(3.10), we obtain

$$
\begin{aligned}
& \int_{\Omega}\left|\partial_{t} \widetilde{y}_{\ell}(\theta, x)\right|^{2} \mathrm{e}^{2 s \varphi(\theta, x)} \mathrm{d} x \\
& \leq C_{3}\left\{\sum_{i, j=1}^{n} \sum_{|\alpha| \leq 1} \int_{Q_{1}} \chi^{2}\left|\partial_{x}^{\alpha} f_{i j}(x)\right|^{2} \mathrm{e}^{2 s \varphi} \mathrm{d} x \mathrm{~d} t+U^{2} \mathrm{e}^{2 s\left(d_{0}-2 \varepsilon\right)}+s \mathrm{e}^{2 s \Phi} V^{2}\right\}
\end{aligned}
$$

for sufficiently large $s>0$. Similarly we have

$$
\begin{aligned}
& \int_{\Omega}\left|\nabla \partial_{t} \widetilde{y}_{\ell}(\theta, x)\right|^{2} \mathrm{e}^{2 s \varphi(\theta, x)} \mathrm{d} x \\
& \leq C_{4}\left\{\sum_{i, j=1}^{n} \sum_{|\alpha| \leq 1} \int_{Q_{1}} \chi^{2}\left|\partial_{x}^{\alpha} f_{i j}(x)\right|^{2} \mathrm{e}^{2 s \varphi} \mathrm{d} x \mathrm{~d} t+U^{2} \mathrm{e}^{2 s\left(d_{0}-2 \varepsilon\right)}+s \mathrm{e}^{2 s \Phi} V^{2}\right\}
\end{aligned}
$$

for sufficiently large $s>0$. By (3.3), we have

$$
L^{(1)} \widetilde{y}_{\ell}(\theta, x)=\sum_{i, j=1}^{n}\left(\partial_{j} f_{i j}(x)\right) \partial_{i} R_{\ell}(\theta, x)+\sum_{i, j=1}^{n} f_{i j}(x) \partial_{i} \partial_{j} R_{\ell}(\theta, x), \quad x \in \Omega
$$

for $1 \leq \ell \leq \frac{(n+1)^{2} n}{2}$. Let us consider the above equations for $1 \leq \ell \leq n+1$. Then we have

$$
\begin{gathered}
\left(\begin{array}{cccc}
\partial_{1} R_{1}(\theta, x) & \partial_{2} R_{1}(\theta, x) & \ldots & \partial_{n} R_{1}(\theta, x) \\
\partial_{1} R_{2}(\theta, x) & \partial_{2} R_{2}(\theta, x) & \ldots & \partial_{n} R_{2}(\theta, x) \\
\vdots & \vdots & \ddots & \vdots \\
\partial_{1} R_{n+1}(\theta, x) & \partial_{2} R_{n+1}(\theta, x) & \ldots & \partial_{n} R_{n+1}(\theta, x)
\end{array}\right)\left(\begin{array}{c}
\sum_{j=1}^{n} \partial_{j} f_{1 j}(x) \\
\sum_{j=1}^{n} \partial_{j} f_{2 j}(x) \\
\vdots \\
\sum_{j=1}^{n} \partial_{j} f_{n j}(x)
\end{array}\right) \\
=\left(\begin{array}{c}
L^{(1)} \widetilde{y}_{1}(\theta, x)-\sum_{i, j=1}^{n} f_{i j} \partial_{i} \partial_{j} R_{1}(\theta, x) \\
L^{(1)} \widetilde{y}_{2}(\theta, x)-\sum_{i, j=1}^{n} f_{i j} \partial_{i} \partial_{j} R_{2}(\theta, x) \\
\vdots \\
L^{(1)} \widetilde{y}_{n+1}(\theta, x)-\sum_{i, j=1}^{n} f_{i j} \partial_{i} \partial_{j} R_{n+1}(\theta, x)
\end{array}\right) .
\end{gathered}
$$


Because linear system (3.14) is composed of $(n+1)$ equations with respect to $n$ unknowns and possesses a solution $\left(\sum_{j=1}^{n} \partial_{j} f_{1 j}(x), \sum_{j=1}^{n} \partial_{j} f_{2 j}(x), \ldots, \sum_{j=1}^{n} \partial_{j} f_{n j}(x)\right)$, the coefficients matrix must satisfy

$$
\operatorname{det}\left(\begin{array}{cccc}
L^{(1)} \widetilde{y}_{1}(\theta, x)-\sum_{i, j=1}^{n} f_{i j} \partial_{i} \partial_{j} R_{1}(\theta, x) & \partial_{1} R_{1}(\theta, x) & \ldots & \partial_{n} R_{1}(\theta, x) \\
L^{(1)} \widetilde{y}_{2}(\theta, x)-\sum_{i, j=1}^{n} f_{i j} \partial_{i} \partial_{j} R_{2}(\theta, x) & \partial_{1} R_{2}(\theta, x) & \ldots & \partial_{n} R_{2}(\theta, x) \\
\vdots & \vdots & \ddots & \vdots \\
L^{(1)} \widetilde{y}_{n+1}(\theta, x)-\sum_{i, j=1}^{n} f_{i j} \partial_{i} \partial_{j} R_{n+1}(\theta, x) & \partial_{1} R_{n+1}(\theta, x) & \ldots & \partial_{n} R_{n+1}(\theta, x)
\end{array}\right)=0
$$

Let us set $D_{i j}^{k}(x) \equiv D_{i j}^{k}\left(\left\{y\left(\left\{a_{i j}^{(2)}\right\}, h_{\ell}\right)\right\}\right)(\theta, x)$. Then we have

$$
\sum_{j=1}^{n} D_{j j}^{1}(x) f_{j j}(x)+2 \sum_{i<j} D_{i j}^{1}(x) f_{i j}(x)=Y_{1}(x), \quad x \in \overline{\Omega \backslash \omega_{1}},
$$

where

We set

$$
Y_{1}(x)=\operatorname{det}\left(\begin{array}{cccc}
L^{(1)} \widetilde{y}_{1}(\theta, x) & \partial_{1} y\left(\left\{a_{i j}^{(2)}\right\}, h_{1}\right)(\theta, x) & \ldots & \partial_{n} y\left(\left\{a_{i j}^{(2)}\right\}, h_{1}\right)(\theta, x) \\
L^{(1)} \widetilde{y}_{2}(\theta, x) & \partial_{1} y\left(\left\{a_{i j}^{(2)}\right\}, h_{2}\right)(\theta, x) & \ldots & \partial_{n} y\left(\left\{a_{i j}^{(2)}\right\}, h_{2}\right)(\theta, x) \\
\vdots & \vdots & \ddots & \vdots \\
L^{(1)} \widetilde{y}_{n+1}(\theta, x) & \partial_{1} y\left(\left\{a_{i j}^{(2)}\right\}, h_{n+1}\right)(\theta, x) & \ldots & \partial_{n} y\left(\left\{a_{i j}^{(2)}\right\}, h_{n+1}\right)(\theta, x)
\end{array}\right)
$$

$$
\begin{aligned}
& Y_{2}(x) \\
& =\operatorname{det}\left(\begin{array}{cccc}
L^{(1)} \widetilde{y}_{n+2}(\theta, x) & \partial_{1} y\left(\left\{a_{i j}^{(2)}\right\}, h_{n+2}\right)(\theta, x) & \ldots & \partial_{n} y\left(\left\{a_{i j}^{(2)}\right\}, h_{n+2}\right)(\theta, x) \\
L^{(1)} \widetilde{y}_{n+3}(\theta, x) & \partial_{1} y\left(\left\{a_{i j}^{(2)}\right\}, h_{n+3}\right)(\theta, x) & \ldots & \partial_{n} y\left(\left\{a_{i j}^{(2)}\right\}, h_{n+3}\right)(\theta, x) \\
\vdots & \vdots & \ddots & \vdots \\
L^{(1)} \widetilde{y}_{2 n+2}(\theta, x) & \partial_{1} y\left(\left\{a_{i j}^{(2)}\right\}, h_{2 n+2}\right)(\theta, x) & \ldots & \partial_{n} y\left(\left\{a_{i j}^{(2)}\right\}, h_{2 n+2}\right)(\theta, x)
\end{array}\right) \text {, } \\
& Y_{\frac{(n+1) n}{2}}(x) \\
& =\operatorname{det}\left(\begin{array}{ccc}
L^{(1)} \widetilde{y}_{\frac{1}{2}(n+1)^{2} n-n}(\theta, x) & \partial_{1} y\left(\left\{a_{i j}^{(2)}\right\}, h_{\frac{1}{2}(n+1)^{2} n-n}\right)(\theta, x) & \ldots \\
L^{(1)} \widetilde{y}_{\frac{1}{2}(n+1)^{2} n-n+1}(\theta, x) & \partial_{1} y\left(\left\{a_{i j}^{(2)}\right\} ; h_{\frac{1}{2}(n+1)^{2} n-n+1}\right)(\theta, x) & \ldots \\
\vdots & \vdots & \ddots \\
L^{(1)} \widetilde{y}_{\frac{1}{2}(n+1)^{2} n}(\theta, x) & \partial_{1} y\left(\left\{a_{i j}^{(2)}\right\} ; h_{\frac{1}{2}(n+1)^{2} n}\right)(\theta, x) & \ldots
\end{array}\right. \\
& \left.\begin{array}{c}
\partial_{n} y\left(\left\{a_{i j}^{(2)}\right\}, h_{\frac{1}{2}(n+1)^{2} n-n}\right)(\theta, x) \\
\partial_{n} y\left(\left\{a_{i j}^{(2)}\right\}, h_{\frac{1}{2}(n+1)^{2} n-n+1}\right)(\theta, x) \\
\vdots \\
\partial_{n} y\left(\left\{a_{i j}^{(2)}\right\}, h_{\frac{1}{2}(n+1)^{2} n}\right)(\theta, x)
\end{array}\right) .
\end{aligned}
$$

Similarly to (3.15), we can obtain

$$
\sum_{j=1}^{n} D_{j j}^{k}(x) f_{j j}(x)+2 \sum_{i<j} D_{i j}^{k}(x) f_{i j}(x)=Y_{k}(x), \quad x \in \overline{\Omega \backslash \omega_{1}}
$$


for $1 \leq k \leq \frac{n(n+1)}{2}$. Equation (3.16) is a linear system with respect to $\frac{n(n+1)}{2}$ unknown $f_{i j}$. Condition (1.7) implies that the determinant of the coefficient matrix does not vanish on $\overline{\Omega \backslash \omega_{1}}$. Applying the Cramer formula, we can solve (3.16) uniquely with respect to $\frac{n(n+1)}{2}$ unknowns $f_{i j}$. Therefore, taking into consideration the definition of $Y_{k}(x)$, we can represent the solution $f_{i j}$ by

$$
f_{i j}(x)=\sum_{\ell=1}^{\frac{(n+1)^{2} n}{2}} c_{i j}^{\ell}(x) L^{(1)} \widetilde{y}_{\ell}(\theta, x), \quad x \in \overline{\Omega \backslash \omega_{1}}, \quad 1 \leq i, j \leq n
$$

with some $c_{i j}^{\ell}(x), 1 \leq i, j \leq n, 1 \leq \ell \leq \frac{(n+1)^{2} n}{2}$. By the Sobolev embedding theorem (e.g., Thm. 5.4 in [1], Cor. 9.1, p. 46, in Vol. 1 of [35]), we see that $H^{m}(\Omega) \subset C^{3}(\bar{\Omega})$. Hence $y\left(\left\{a_{i j}^{(2)}\right\}, h_{\ell}\right) \in C^{3}\left(\left[\tau_{1}, \tau_{2}\right] ; H^{m}(\Omega)\right) \subset$ $C^{3}\left(\left[\tau_{1}, \tau_{2}\right] ; C^{3}(\bar{\Omega})\right)$, and so $c_{i j}^{\ell} \in C^{1}\left(\overline{\Omega \backslash \omega_{1}}\right), 1 \leq i, j \leq n, 1 \leq \ell \leq \frac{(n+1)^{2} n}{2}$. Moreover, since $c_{i j}^{\ell}$ are given by values (not including the derivatives) of $D_{i j}^{k}(x)$, we see that $\left\|c_{i j}^{\ell}\right\|_{C^{1}\left(\overline{\Omega \backslash \omega_{1}}\right)} \leq C_{5}^{\prime}$.

By noting also that $f_{i j}(x)=0, x \in \omega_{1}, 1 \leq i, j \leq n$, by means of (3.17) and $c_{i j}^{\ell} \in C^{1}\left(\overline{\Omega \backslash \omega_{1}}\right)$, we have

$$
\begin{aligned}
& \int_{\Omega} \sum_{|\alpha| \leq 1}\left|\partial_{x}^{\alpha} f_{i j}(x)\right|^{2} \mathrm{e}^{2 s \varphi(\theta, x)} \mathrm{d} x \leq C_{5} \sum_{\ell=1}^{\frac{(n+1)^{2} n}{2}} \sum_{|\alpha| \leq 1} \int_{\Omega}\left|\partial_{x}^{\alpha} \partial_{t} \widetilde{y}_{\ell}(\theta, x)\right|^{2} \mathrm{e}^{2 s \varphi(\theta, x)} \mathrm{d} x \\
& +C_{5} \sum_{\ell=1}^{\frac{(n+1)^{2} n}{2}} \sum_{|\alpha| \leq 3} \int_{\Omega}\left|\partial_{x}^{\alpha} \widetilde{y}_{\ell}(\theta, x)\right|^{2} \mathrm{e}^{2 s \varphi(\theta, x)} \mathrm{d} x, \quad 1 \leq i, j \leq n, \quad 1 \leq \ell \leq \frac{(n+1)^{2} n}{2} .
\end{aligned}
$$

By (3.11) and (3.12), we have

$$
\begin{aligned}
& \sum_{\ell=1}^{\frac{(n+1)^{2} n}{2}} \sum_{|\alpha| \leq 1} \int_{\Omega}\left|\partial_{x}^{\alpha} \partial_{t} \widetilde{y}_{\ell}(\theta, x)\right|^{2} \mathrm{e}^{2 s \varphi(\theta, x)} \mathrm{d} x \\
& \leq C_{6} \sum_{i, j=1}^{n} \sum_{|\alpha| \leq 1} \int_{\Omega}\left|\partial_{x}^{\alpha} f_{i j}(x)\right|^{2} \mathrm{e}^{2 s \varphi(\theta, x)}\left(\int_{\tau_{1}}^{\tau_{2}} \mathrm{e}^{2 s(\varphi(t, x)-\varphi(\theta, x))} \mathrm{d} t\right) \mathrm{d} x \\
& +C_{6} U^{2} \mathrm{e}^{2 s\left(d_{0}-2 \varepsilon\right)}+C_{6} s \mathrm{e}^{2 s \Phi} V^{2}
\end{aligned}
$$

for all large $s>0$. By (3.18) and (3.19), we obtain

$$
\begin{aligned}
& \sum_{i, j=1}^{n} \sum_{|\alpha| \leq 1} \int_{\Omega}\left|\partial_{x}^{\alpha} f_{i j}(x)\right|^{2} \mathrm{e}^{2 s \varphi(\theta, x)} \mathrm{d} x \\
& \leq C_{7} \sum_{i, j=1}^{n} \sum_{|\alpha| \leq 1} \int_{\Omega}\left|\partial_{x}^{\alpha} f_{i j}(x)\right|^{2} \mathrm{e}^{2 s \varphi(\theta, x)}\left(\int_{\tau_{1}}^{\tau_{2}} \mathrm{e}^{2 s(\varphi(t, x)-\varphi(\theta, x))} \mathrm{d} t\right) \mathrm{d} x \\
& +C_{7} \sum_{\ell=1}^{\frac{(n+1)^{2} n}{2}} \sum_{|\alpha| \leq 3} \int_{\Omega}\left|\partial_{x}^{\alpha} \widetilde{y}_{\ell}(\theta, x)\right|^{2} \mathrm{e}^{2 s \varphi(\theta, x)} \mathrm{d} x+C_{7} U^{2} \mathrm{e}^{2 s\left(d_{0}-2 \varepsilon\right)}+C_{7} s \mathrm{e}^{2 s \Phi} V^{2}
\end{aligned}
$$


for large $s>0$. Applying the Lebesgue theorem, we have

$$
\begin{aligned}
& \quad \sup _{x \in \Omega}\left|\int_{\tau_{1}}^{\tau_{2}} \mathrm{e}^{2 s(\varphi(t, x)-\varphi(\theta, x))} \mathrm{d} t\right|=\sup _{x \in \Omega}\left|\int_{\tau_{1}}^{\tau_{2}} \exp \left(2 s \mathrm{e}^{\lambda d(x)}\left(\mathrm{e}^{-\lambda \beta|t-\theta|^{2}}-1\right)\right) \mathrm{d} t\right| \\
& \leq \int_{\tau_{1}}^{\tau_{2}} \exp \left(2 s \mathrm{e}^{\lambda d_{1}}\left(\mathrm{e}^{-\lambda \beta|t-\theta|^{2}}-1\right)\right) \mathrm{d} t=o(1) \quad \text { as } s \longrightarrow \infty,
\end{aligned}
$$

where $d_{1}=\inf _{x \in \Omega} d(x)$. Then

$$
\begin{aligned}
& \sum_{i, j=1}^{n} \sum_{|\alpha| \leq 1} \int_{\Omega}\left|\partial_{x}^{\alpha} f_{i j}(x)\right|^{2} \mathrm{e}^{2 s \varphi(\theta, x)}\left(\int_{\tau_{1}}^{\tau_{2}} \mathrm{e}^{2 s(\varphi(t, x)-\varphi(\theta, x))} \mathrm{d} t\right) \mathrm{d} x \\
& =o(1) \sum_{i, j=1}^{n} \sum_{|\alpha| \leq 1} \int_{\Omega}\left|\partial_{x}^{\alpha} f_{i j}(x)\right|^{2} \mathrm{e}^{2 s \varphi(\theta, x)} \mathrm{d} x \quad \text { as } s \longrightarrow \infty
\end{aligned}
$$

Hence, from (3.20) we have

$$
\begin{aligned}
& (1-o(1)) \sum_{i, j=1}^{n} \sum_{|\alpha| \leq 1} \int_{\Omega}\left|\partial_{x}^{\alpha} f_{i j}(x)\right|^{2} \mathrm{e}^{2 s \varphi(\theta, x)} \mathrm{d} x \leq C_{8} U^{2} \mathrm{e}^{2 s\left(d_{0}-2 \varepsilon\right)}+C_{8} s \mathrm{e}^{2 s \Phi} V^{2} \\
& +C_{8} \sum_{\ell=1}^{\frac{(n+1)^{2} n}{2}} \sum_{|\alpha| \leq 3} \int_{\Omega}\left|\partial_{x}^{\alpha} \widetilde{y}_{\ell}(\theta, x)\right|^{2} \mathrm{e}^{2 s \varphi(\theta, x)} \mathrm{d} x \quad \text { as } s \longrightarrow \infty .
\end{aligned}
$$

By $\varphi(\theta, x) \geq d_{0}$ for $x \in \bar{\Omega}$, we obtain

$$
\begin{aligned}
& (1-o(1)) \sum_{i, j=1}^{n} \sum_{|\alpha| \leq 1} \mathrm{e}^{2 s d_{0}} \int_{\Omega}\left|\partial_{x}^{\alpha} f_{i j}(x)\right|^{2} \mathrm{~d} x \leq C_{9} U^{2} \mathrm{e}^{2 s\left(d_{0}-2 \varepsilon\right)}+C_{9} s \mathrm{e}^{2 s \Phi} V^{2} \\
& +C_{9} \sum_{\ell=1}^{\frac{(n+1)^{2} n}{2}} \sum_{|\alpha| \leq 3} \int_{\Omega}\left|\partial_{x}^{\alpha} \widetilde{y}_{\ell}(\theta, x)\right|^{2} \mathrm{e}^{2 s \varphi(\theta, x)} \mathrm{d} x \quad \text { as } s \longrightarrow \infty,
\end{aligned}
$$

that is,

$$
\begin{aligned}
& (1-o(1)) \sum_{i, j=1}^{n} \sum_{|\alpha| \leq 1} \int_{\Omega}\left|\partial_{x}^{\alpha} f_{i j}(x)\right|^{2} \mathrm{~d} x \leq C_{9} U^{2} \mathrm{e}^{-4 s \varepsilon}+C_{9} s \mathrm{e}^{2 s\left(\Phi-d_{0}\right)} V^{2} \\
& +C_{9} \sum_{\ell=1}^{\frac{(n+1)^{2} n}{2}} \sum_{|\alpha| \leq 3} \int_{\Omega}\left|\partial_{x}^{\alpha} \widetilde{y}_{\ell}(\theta, x)\right|^{2} \mathrm{e}^{2 s\left(\Phi-d_{0}\right)} \mathrm{d} x \quad \text { as } s \longrightarrow \infty .
\end{aligned}
$$

On the other hand, we can prove the following estimate:

$$
U^{2} \leq C_{10} V^{2}+C_{10} \sum_{i, j=1}^{n} \sum_{|\alpha| \leq 1} \int_{\Omega}\left|\partial_{x}^{\alpha} f_{i j}(x)\right|^{2} \mathrm{~d} x .
$$


In fact, by (3.3) and (3.4) we have

$$
\left\{\begin{array}{l}
L^{(1)} \partial_{t} \widetilde{y}_{\ell}(t, x)=\sum_{i, j=1}^{n} \partial_{j}\left(f_{i j}(x) \partial_{i} \partial_{t} R_{\ell}(t, x)\right), \quad(t, x) \in(0, T) \times \Omega \\
\partial_{t} \widetilde{y}_{\ell}(t, x)=0, \quad(t, x) \in(0, T) \times \partial \Omega, 1 \leq \ell \leq \frac{(n+1)^{2} n}{2}
\end{array}\right.
$$

Apply Lemma 1.1 in [19] ( $c f$. Lem. 2.4 in [20]) to (3.23). Then we can see that there exist $\psi_{0} \in C^{2}(\bar{\Omega})$ and a constant $\sigma_{0}>0$ such that for a constant $\sigma \geq \sigma_{0}$ we can choose $\eta_{0}(\sigma)>0$ such that for each $\eta \geq \eta_{0}(\sigma)$, we have

$$
\begin{aligned}
& \int_{\left(\tau_{3}, \tau_{4}\right) \times \Omega}\left(\frac{\eta \mathrm{e}^{\sigma \psi_{0}(x)}}{\left(t-\tau_{3}\right)\left(\tau_{4}-t\right)}\left|\nabla \partial_{t} \widetilde{y}_{\ell}\right|^{2}+\frac{\eta^{3} \mathrm{e}^{3 \sigma \psi_{0}(x)}}{\left(t-\tau_{3}\right)^{3}\left(\tau_{4}-t\right)^{3}}\left|\partial_{t} \widetilde{y}_{\ell}\right|^{2}\right) J(t, x) \mathrm{d} x \mathrm{~d} t \\
& \leq C_{11} \sum_{i, j=1}^{n} \sum_{|\alpha|=1} \int_{\left(\tau_{3}, \tau_{4}\right) \times \Omega}\left|\partial_{x}^{\alpha} f_{i j}(x)\right|^{2} J(t, x) \mathrm{d} x \mathrm{~d} t \\
& +C_{11} \eta \int_{\tau_{3}}^{\tau_{4}} \int_{\Gamma_{0}}\left|\partial_{\nu} \partial_{t} \widetilde{y}_{\ell}\right|^{2} \frac{\eta \mathrm{e}^{\sigma \psi_{0}(x)}}{\left(t-\tau_{3}\right)\left(\tau_{4}-t\right)} J(t, x) \mathrm{d} \Sigma .
\end{aligned}
$$

Here we set

$$
J(t, x)=\exp \left\{\frac{2 \eta\left(\mathrm{e}^{\sigma \psi_{0}(x)}-\mathrm{e}^{\left.2 \sigma\left\|\psi_{0}\right\|_{C(\bar{\Omega})}\right)}\right.}{\left(t-\tau_{3}\right)\left(\tau_{4}-t\right)}\right\} .
$$

By the proof of Lemma 1.1 in [19], we see that the constant $C_{11}>0$ can be taken uniformly in $a_{i j} \in \mathcal{U}$, and see also $[9,15]$ as for the proof. We note that $C_{11}$ is dependent on $\sigma$, but independent of $\eta$, and the constant $\sigma_{0}$ depends on $\mathcal{U}$. We fix $\sigma>\sigma_{0}$ and $\eta>\eta_{0}(\sigma)$. Then

$$
0<C_{11} \leq J(t, x)
$$

for $x \in \bar{\Omega}$ and $\tau_{1}<t<\tau_{2}$ and

$$
J(t, x), \quad \frac{\eta \mathrm{e}^{\sigma \psi_{0}(x)}}{\left(t-\tau_{3}\right)\left(\tau_{4}-t\right)} J(t, x) \leq C_{12}
$$

for $x \in \bar{\Omega}$ and $\tau_{3}<t<\tau_{4}$. Hence we have

$$
\begin{aligned}
& \int_{Q_{1}}\left(\left|\nabla \partial_{t} \widetilde{y}_{\ell}\right|^{2}+\left|\partial_{t} \widetilde{y}_{\ell}\right|^{2}\right) \mathrm{d} x \mathrm{~d} t \\
& \leq C_{13} \sum_{i, j=1}^{n} \sum_{|\alpha| \leq 1} \int_{\left(\tau_{3}, \tau_{4}\right) \times \Omega}\left|\partial_{x}^{\alpha} f_{i j}(x)\right|^{2} \mathrm{~d} x \mathrm{~d} t+C_{13} \int_{\tau_{3}}^{\tau_{4}} \int_{\Gamma_{0}}\left|\partial_{\nu} \partial_{t} \widetilde{y}_{\ell}\right|^{2} \mathrm{~d} \Sigma .
\end{aligned}
$$

Similarly, we can obtain

$$
\begin{aligned}
& \int_{Q_{1}}\left(\left|\nabla \partial_{t}^{2} \widetilde{y}_{\ell}\right|^{2}+\left|\partial_{t}^{2} \widetilde{y}_{\ell}\right|^{2}\right) \mathrm{d} x \mathrm{~d} t \\
& \leq C_{13} \sum_{i, j=1}^{n} \sum_{|\alpha| \leq 1} \int_{\left(\tau_{3}, \tau_{4}\right) \times \Omega}\left|\partial_{x}^{\alpha} f_{i j}(x)\right|^{2} \mathrm{~d} x \mathrm{~d} t+C_{13} \int_{\tau_{3}}^{\tau_{4}} \int_{\Gamma_{0}}\left|\partial_{\nu} \partial_{t}^{2} \widetilde{y}_{\ell}\right|^{2} \mathrm{~d} \Sigma
\end{aligned}
$$

By (3.24) and (3.25), we complete the proof of (3.22).

We can obtain (1.6) by substituting (3.22) into (3.21) and taking $s$ large enough. Thus the proof of Theorem 1.1 is completed. 
Proof of Theorem 1.2. Let $B=\left(b_{i j}\right)_{1 \leq i, j \leq n}$ be an $n \times n$ matrix such that $b_{i j} \in \mathbb{R}$ and $\operatorname{det} B>0$. We set

$$
\widetilde{g}_{i}(x)=\sum_{j=1}^{n} b_{i j} x_{j}, \quad 1 \leq i \leq n
$$

and

$$
\begin{aligned}
& \widehat{g}_{1}(x)=x_{1}^{2}, \widehat{g}_{2}(x)=2 x_{1} x_{2}, \widehat{g}_{3}(x)=2 x_{1} x_{3}, \ldots, \widehat{g}_{n}(x)=2 x_{1} x_{n}, \\
& \widehat{g}_{n+1}(x)=x_{2}^{2}, \widehat{g}_{n+2}(x)=2 x_{2} x_{3}, \ldots, \widehat{g}_{2 n-1}(x)=2 x_{2} x_{n}, \\
& \vdots \quad \vdots \\
& \widehat{g}_{\frac{(n+1) n}{2}-2}(x)=x_{n-1}^{2}, \widehat{g}_{\frac{(n+1) n}{2}-1}(x)=2 x_{n-1} x_{n}, \\
& \widehat{g}_{\frac{(n+1) n}{2}}(x)=x_{n}^{2} .
\end{aligned}
$$

Let us define an $\frac{(n+1)^{2} n}{2}$-dimensional vector by

$$
\begin{aligned}
& \left(g_{1}(x), g_{2}(x), \ldots, g_{n+1}(x), g_{n+2}(x), g_{n+3}(x), \ldots, g_{2 n+2}(x), \ldots,\right. \\
& \left.g_{\frac{n^{3}+2 n^{2}-n}{2}}(x), g_{\frac{n^{3}+2 n^{2}-n}{2}+1}(x), \ldots, g_{\frac{n^{3}+2 n^{2}-n}{2}+n}(x)\right) \\
= & \left(\widehat{g}_{1}(x), \widetilde{g}_{1}(x), \ldots, \widetilde{g}_{n}(x), \widehat{g}_{2}(x), \widetilde{g}_{1}(x), \ldots, \widetilde{g}_{n}(x), \ldots,\right. \\
& \left.\widehat{g}_{\frac{n(n+1)}{2}}(x), \widetilde{g}_{1}(x), \ldots, \widetilde{g}_{n}(x)\right)
\end{aligned}
$$

Therefore, noting that $\partial_{i} \partial_{j} \widetilde{g_{k}}=0$, we obtain

$$
\begin{aligned}
D_{i j}^{k}\left(\left\{g_{\ell}\right\}\right)(x) & =\operatorname{det}\left(\begin{array}{ll}
\partial_{i} \partial_{j} \widehat{g}_{k} & * \\
0 & B
\end{array}\right) \\
& =\left(\partial_{i} \partial_{j} \widehat{g}_{k}\right) \operatorname{det} B, \quad 1 \leq k \leq \frac{n(n+1)}{2}, 1 \leq i, j \leq n .
\end{aligned}
$$

Hence

$$
D\left(\left\{g_{\ell}\right\}\right)(x)=\left(\begin{array}{cccccc}
2 \operatorname{det} B & 0 & 0 & \cdots & 0 & 0 \\
0 & 2 \operatorname{det} B & 0 & \cdots & 0 & 0 \\
0 & 0 & 2 \operatorname{det} B & \cdots & 0 & 0 \\
\vdots & \vdots & \vdots & \ddots & \vdots & \vdots \\
0 & 0 & 0 & \cdots & 2 \operatorname{det} B & 0 \\
0 & 0 & 0 & \cdots & 0 & 2 \operatorname{det} B
\end{array}\right) .
$$

Consequently we have $\operatorname{det} D\left(\left\{g_{\ell}\right\}\right)(x)=(2 \operatorname{det} B)^{\frac{(n+1) n}{2}}>0$. We introduce a cut-off function $\chi_{1} \in C_{0}^{\infty}(\Omega)$ such that $\chi_{1}=1$ on $\overline{\Omega \backslash \omega_{1}}$. Then we have

$$
\chi_{1} g_{\ell} \in \mathcal{D}\left(A^{\left[\frac{m+1}{2}\right]}\right) \text { and } D\left(\left\{\chi_{1} g_{\ell}\right\}\right)(x)>0, \quad x \in \overline{\Omega \backslash \omega_{1}}
$$

Here we recall that $A$ is defined by (1.9). 
By Proposition 1.1, for arbitrarily $\mu_{\ell} \in L^{2}(\Omega)$, we can choose $h_{\ell} \in C_{0}^{\infty}((0, T) \times \omega), 1 \leq \ell \leq \frac{(n+3) n}{2}$, so that for a sufficiently small $\varepsilon>0$ we have

$$
\left\|y\left(\left\{a_{i j}^{(2)}\right\}, h_{\ell}, \mu_{\ell}\right)(\theta, \cdot)-\chi_{1} \widehat{g}_{\ell}\right\|_{H^{m}(\Omega)} \leq \varepsilon, \quad 1 \leq \ell \leq \frac{(n+1) n}{2}
$$

and

$$
\left\|y\left(\left\{a_{i j}^{(2)}\right\}, h_{\frac{(n+1) n}{2}+k}, \mu_{\frac{(n+1) n}{2}+k}\right)(\theta, \cdot)-\chi_{1} \widetilde{g}_{k}\right\|_{H^{m}(\Omega)} \leq \varepsilon, \quad 1 \leq k \leq n .
$$

Here we note that $y\left(\left\{a_{i j}^{(2)}\right\}, h, \mu\right)$ denotes the solution to (1.1) and (1.2) with $y(0, \cdot)=\mu$. Since $m>\frac{n}{2}+3$, we have $H^{m}(\Omega) \subset C^{2}(\bar{\Omega})$. Then we can obtain

$$
\left\|y\left(\left\{a_{i j}^{(2)}\right\}, h_{\ell}, \mu_{\ell}\right)(\theta, \cdot)-\chi_{1} \widehat{g}_{\ell}\right\|_{C^{2}\left(\overline{\Omega \backslash \omega_{1}}\right)} \leq \varepsilon, \quad 1 \leq \ell \leq \frac{(n+1) n}{2}
$$

and

Let

$$
\left\|y\left(\left\{a_{i j}^{(2)}\right\}, h_{\frac{(n+1) n}{2}+k}, \mu_{\frac{(n+1) n}{2}+k}\right)(\theta, \cdot)-\chi_{1} \widetilde{g}_{k}\right\|_{C^{2}\left(\overline{\Omega \backslash \omega_{1}}\right)} \leq \varepsilon, \quad 1 \leq k \leq n .
$$

$$
\begin{aligned}
& \left(\widehat{h}_{\ell}\right)_{1 \leq \ell \leq \frac{(n+1)^{2} n}{2}} \\
& =\left(\widehat{h}_{1}, \widehat{h}_{2}, \ldots, \widehat{h}_{n+1}, \widehat{h}_{n+2}, \widehat{h}_{n+3}, \ldots, \widehat{h}_{2 n+2}, \ldots,\right. \\
& \left.\widehat{h}_{\frac{n^{3}+2 n^{2}-n}{2}}, \widehat{h}_{\frac{n^{3}+2 n^{2}-n}{2}+1}, \ldots, \widehat{h}_{\frac{n^{3}+2 n^{2}-n}{2}+n}\right) \\
& \equiv\left(h_{1}, h_{\frac{n(n+1)}{2}+1}, \ldots, h_{\frac{n(n+1)}{2}+n}, h_{2}, h_{\frac{n(n+1)}{2}+1}, \ldots, h_{\frac{n(n+1)}{2}+n}, \ldots,\right. \\
& \left.h_{\frac{n(n+1)}{2}}, h_{\frac{n(n+1)}{2}+1}, \ldots, h_{\frac{n(n+1)}{2}+n}\right)
\end{aligned}
$$

and

$$
\begin{aligned}
& \left(\widehat{\mu}_{\ell}\right)_{1 \leq \ell \leq \frac{(n+1)^{2} n}{2}} \equiv\left(\widehat{\mu}_{1}, \widehat{\mu}_{2}, \ldots, \widehat{\mu}_{n+1}, \widehat{\mu}_{n+2}, \widehat{\mu}_{n+3}, \ldots, \widehat{\mu}_{2 n+2}, \ldots,\right. \\
& \widehat{\mu}_{\frac{n^{3}+2 n^{2}-n}{2}}, \widehat{\mu}_{\frac{n^{3}+2 n^{2}-n}{2}}+1 \\
& =\left(\mu_{1}, \mu_{\frac{n(n+1)}{2}+1}, \ldots, \widehat{\mu}_{\frac{n^{3}+2 n^{2}-n}{2}+n}\right) \\
& \left.\mu_{\frac{n(n+1)}{2}+n}, \mu_{\frac{n(n+1)}{2}+1}, \ldots, \mu_{\frac{n(n+1)}{2}+n}\right) .
\end{aligned}
$$

By (3.27)-(3.31), we can obtain

$$
D\left(\left\{y\left(\left\{a_{i j}^{(2)}\right\}, \widehat{h}_{\ell}, \widehat{\mu}_{\ell}\right)\right\}\right)(\theta, x)>0, \quad x \in \overline{\Omega \backslash \omega_{1}}
$$

by taking $\varepsilon$ small enough. Thus, by applying Theorem 1.1 to $\widehat{h}_{\ell}, 1 \leq \ell \leq \frac{(n+1)^{2} n}{2}$, the proof of Theorem 1.2 is completed. 


\section{Proof of Proposition 1.1}

For the proof, we will use the dual space $\mathcal{D}\left(A^{-m_{1}}\right)$, which is defined as follows. By $\|\cdot\|$ and $(\cdot, \cdot)$ we denote the norm and the scalar product in $L^{2}(\Omega)$, respectively. We recall that the operator $A$ in $L^{2}(\Omega)$ is defined by (1.9). Henceforth $[\gamma]$ denotes the greatest integer not exceeding $\gamma \in \mathbb{R}$. Since $a_{i j} \in C^{m-1,1}(\bar{\Omega})$, elliptic regularity results (e.g., Thm. 8.13, p. 187, in [16]) yield

$$
C_{1}^{-1}\|A u\| \leq\|u\|_{H^{2}(\Omega)} \leq\|A u\|, \quad u \in \mathcal{D}(A) .
$$

Next, using $\left\|A^{k-1} u\right\| \leq C_{1}^{\prime}\left\|A^{k} u\right\|$ for $u \in \mathcal{D}\left(A^{k}\right)$ and $k \in \mathbb{N}$, we repeatedly apply Theorem 8.13 in [16] and we see

$$
C_{1}^{-1}\left\|A^{k} u\right\| \leq\|u\|_{H^{2 k}(\Omega)} \leq C_{1}\left\|A^{k} u\right\|, \quad u \in \mathcal{D}\left(A^{k}\right)
$$

where $k=0,1, \ldots,\left[\frac{m+1}{2}\right]$. Here the constant $C_{1}>0$ is independent of $u \in \mathcal{D}\left(A^{k}\right)$, and $\|\cdot\|_{H^{2 k}(\Omega)}$ denotes the norm in $H^{2 k}(\Omega)$. In particular,

$$
C_{1}^{-1}\left\|A^{m_{1}} u\right\| \leq\|u\|_{H^{2 m_{1}}(\Omega)} \leq C_{1}\left\|A^{m_{1}} u\right\|, \quad u \in \mathcal{D}\left(A^{m_{1}}\right)
$$

Moreover it is known that there exists a sequence of eigenvalues $\left\{\kappa_{j}\right\}_{j \in \mathbb{N}}$ of $A$ :

$$
0<\kappa_{1} \leq \kappa_{2} \leq \ldots \longrightarrow \infty
$$

where $\kappa_{j}$ appears the same time as its multiplicity. Then we can form an orthonormal basis $\left\{e_{j}\right\}_{j \in \mathbb{N}}$ in $L^{2}(\Omega)$ such that $A e_{j}=\kappa_{j} e_{j}$. We have

$$
\left\|A^{\ell} u\right\|=\left(\sum_{j=1}^{\infty} \kappa_{j}^{2 \ell}\left(u, e_{j}\right)^{2}\right)^{\frac{1}{2}}
$$

and $\mathcal{D}\left(A^{\ell}\right), \ell \in \mathbb{N} \cup\{0\}$, is a Hilbert space with the scalar product

$$
(u, v)_{\mathcal{D}\left(A^{\ell}\right)}=\sum_{j=1}^{\infty} \kappa_{j}^{2 \ell}\left(u, e_{j}\right)\left(v, e_{j}\right)
$$

In particular, $\mathcal{D}\left(A^{0}\right)=L^{2}(\Omega)$, and $\mathcal{D}\left(A^{m_{1}}\right)$ is dense in $L^{2}(\Omega)$, and the embedding is continuous. Identifying the dual space of $L^{2}(\Omega)$ with $L^{2}(\Omega)$, we have $\mathcal{D}\left(A^{m_{1}}\right) \subset L^{2}(\Omega) \subset\left(\mathcal{D}\left(A^{m_{1}}\right)\right)^{\prime}$ topologically (e.g., [6]). Henceforth we set $\left(\mathcal{D}\left(A^{m_{1}}\right)\right)^{\prime}=\mathcal{D}\left(A^{-m_{1}}\right)$ and $\mathcal{D}\left(A^{m_{1}}\right)<u, \xi>_{\mathcal{D}\left(A^{-m_{1}}\right)}$ denotes the value of a linear functional $\xi \in\left(\mathcal{D}\left(A^{m_{1}}\right)\right)^{\prime}$ at $u$. We note that

$$
\mathcal{D}\left(A^{m_{1}}\right)<u, \xi>_{\mathcal{D}\left(A^{-m_{1}}\right)}=(u, \xi)
$$

if $u \in \mathcal{D}\left(A^{m_{1}}\right)$ and $\xi \in L^{2}(\Omega)(e . g ., \mathrm{V} .2$ in $[6])$.

Since $L^{2}(\Omega)$ is dense in $\mathcal{D}\left(A^{-m_{1}}\right)$, in terms of the choice of the norm on $\mathcal{D}(A)$, we see that $A^{-m_{1}}$ is extended uniquely to a bounded operator in $\mathcal{D}\left(A^{-m_{1}}\right)$ and $\|u\|_{\mathcal{D}\left(A^{-m_{1}}\right)}=\left\|A^{-m_{1}} u\right\|$. By the density of $C_{0}^{\infty}(\Omega)$ in $L^{2}(\Omega)$, we see also that $C_{0}^{\infty}(\Omega)$ is dense in $\mathcal{D}\left(A^{-m_{1}}\right)$. Furthermore it is seen that $\mathrm{e}^{-t A}$ is an analytic semigroup in $\mathcal{D}\left(A^{-m_{1}}\right)$ and $A^{-m_{1}} \mathrm{e}^{-t A}=\mathrm{e}^{-t A} A^{-m_{1}}$.

Now we proceed to the proof of the proposition. Without loss of generality, we can suppose that $y(0)=\mu=0$, because the parabolic equation (1.1) is linear. First we consider

$$
\left\{\begin{array}{l}
-\frac{\partial z}{\partial t}+A z(t, x)=0, \quad(t, x) \in Q \\
z=0, \quad(t, x) \in \Sigma \\
z(T, x)=\xi(x), \quad x \in \Omega
\end{array}\right.
$$


where $\xi \in \mathcal{D}\left(A^{-m_{1}}\right)$. We can verify (e.g., [37]) that for every $\xi \in \mathcal{D}\left(A^{-m_{1}}\right)$, there exists a unique solution $z \in C\left([0, T] ; \mathcal{D}\left(A^{-m_{1}}\right)\right)$ such that

$$
\|z\|_{C\left([0, T] ; \mathcal{D}\left(A^{\left.\left.-m_{1}\right)\right)}\right.\right.} \leq C\|\xi\|_{\mathcal{D}\left(A^{-m_{1}}\right)} .
$$

Recall that $y\left(\left\{a_{i j}\right\}, h, 0\right)(t, x)$ is the solution to (1.1) and (1.2) with $y(0)=0$ where $h \in C_{0}^{\infty}((0, T) \times \omega)$. We will prove

$$
\mathcal{D}\left(A^{m_{1}}\right)\left\langle y\left(\left\{a_{i j}\right\}, h, 0\right)(T, \cdot), \xi\right\rangle_{\mathcal{D}\left(A^{-m_{1}}\right)}{ }_{L^{2}\left(0, T ; \mathcal{D}\left(A^{m_{1}}\right)\right)}\langle h, z\rangle_{L^{2}\left(0, T ; \mathcal{D}\left(A^{-m_{1}}\right)\right)} \cdot
$$

In fact, by the density of $C_{0}^{\infty}(\Omega)$ in $\mathcal{D}\left(A^{-m_{1}}\right)$, there exists a sequence $\xi_{k} \in C_{0}^{\infty}(\Omega), k \in \mathbb{N}$ such that $\xi_{k} \longrightarrow \xi$ in $\mathcal{D}\left(A^{-m_{1}}\right)$. By $z_{k}$ we denote the solution to (4.3) with the final value $\xi_{k}$ at $t=T$. Then $z_{k}, y\left(\left\{a_{i j}\right\}, h, 0\right) \in$ $C^{1}\left([0, T] ; L^{2}(\Omega)\right) \cap C([0, T] ; \mathcal{D}(A))\left(\right.$ e.g. Thm. 3.5 , p. 114, in [37]). Therefore we can multiply $(1.1)$ with $z_{k}(t, x)$, so that by integrating by parts, we have

$$
\left(y\left(\left\{a_{i j}\right\}, h, 0\right)(T, \cdot), \xi_{k}\right)_{L^{2}(\Omega)}=\left(h, z_{k}\right)_{L^{2}((0, T) \times \Omega)} .
$$

Noting that $h \in C_{0}^{\infty}((0, T) \times \omega)$, we can further rewrite it as

$$
\mathcal{D}\left(A^{m_{1}}\right)\left\langle y\left(\left\{a_{i j}\right\}, h, 0\right)(T, \cdot), \xi_{k}\right\rangle_{\mathcal{D}\left(A^{-m_{1}}\right)}={ }_{L^{2}\left(0, T ; \mathcal{D}\left(A^{m_{1}}\right)\right)}\left\langle h, z_{k}\right\rangle_{L^{2}\left(0, T ; \mathcal{D}\left(A^{-m_{1}}\right)\right)} \cdot
$$

Since $y\left(\left\{a_{i j}\right\}, h, 0\right)(t, \cdot)=\int_{0}^{t} \mathrm{e}^{-(t-s) A} h(s, \cdot) \mathrm{d} s$ for $t>0(e . g .,[37])$ and $h \in C_{0}^{\infty}((0, T) \times \omega)$, we directly see that $y\left(\left\{a_{i j}\right\}, h, 0\right)(T, \cdot) \in \mathcal{D}\left(A^{m_{1}}\right)$. Hence, as $k \longrightarrow \infty$, we have

$$
\mathcal{D}\left(A^{m_{1}}\right)\left\langle y\left(\left\{a_{i j}\right\}, h, 0\right)(T, \cdot), \xi\right\rangle_{\mathcal{D}\left(A^{-m_{1}}\right)}={ }_{L^{2}\left(0, T ; \mathcal{D}\left(A^{m_{1}}\right)\right)}\langle h, z\rangle_{L^{2}\left(0, T ; \mathcal{D}\left(A^{-m_{1}}\right)\right)} .
$$

Thus we proved (4.4).

For the proof of the proposition, it is sufficient to verify that if

$$
\mathcal{D}\left(A^{m_{1}}\right)\left\langle y\left(\left\{a_{i j}\right\}, h, 0\right)(T, \cdot), \xi\right\rangle_{\mathcal{D}\left(A^{\left.-m_{1}\right)}\right.}=0
$$

for all $h \in C_{0}^{\infty}((0, T) \times \omega)$, then $\xi=0$. Let us assume (4.5). Then for any $\delta \in(0, T)$, by (4.4) we have

$$
L^{2}\left(0, T-\delta ; \mathcal{D}\left(A^{m_{1}}\right)\right)\langle h, z\rangle_{L^{2}\left(0, T-\delta ; \mathcal{D}\left(A^{-m_{1}}\right)\right)}=0 \quad \text { for all } h \in C_{0}^{\infty}((0, T-\delta) \times \omega) .
$$

By the smoothing property for the parabolic equation (e.g., [37]), we know that $z \in L^{2}(0, T-\delta ; \mathcal{D}(A)) \subset$ $L^{2}\left(0, T-\delta ; H^{2}(\Omega) \cap H_{0}^{1}(\Omega)\right)$. Therefore

$$
\begin{aligned}
& L^{2}\left(0, T-\delta ; \mathcal{D}\left(A^{m_{1}}\right)\right) \\
& =(h, z)_{L^{2}\left(0, T-\delta ; L^{2}(\omega)\right)}
\end{aligned}
$$

for all $h \in C_{0}^{\infty}((0, T-\delta) \times \omega)$. Hence we have $z=0$ in $(0, T-\delta) \times \omega$. By the unique continuation for parabolic equations (e.g., Saut and Scheurer [38]), we can see that $z=0$ in $(0, T-\delta) \times \Omega$. We note that a Carleman estimate yields the unique continuation by an argument similar to the one in obtaining (3.22). Since $\delta$ is arbitrary and $z \in C\left([0, T] ; \mathcal{D}\left(A^{-m_{1}}\right)\right)$, we can obtain $\xi=0$. Thus the proof of Proposition 1.1 is completed. 


\section{Appendix A. Proof of Theorem 2.1}

The proof is adapted from the proofs in $[15,19]$, where the authors treat the case of the weight function containing a singular function.

Henceforth we take $\lambda>1$ and by $C_{j}$ we denote generic constants which do not depend on $s$ and $\lambda$, and continuously depend on $\sum_{i, j=1}^{n}\left\|a_{i j}\right\|_{C^{1}(\bar{\Omega})}, \tau_{1}, \tau_{2}, \Omega, r$. It suffices to prove (2.6) for the operator

$$
\widetilde{L} v=\partial_{t} v-\sum_{i, j=1}^{n} a_{i j}(x) \partial_{i} \partial_{i} v
$$

In fact, we have

$$
L v=\widetilde{L} v-\sum_{i, j=1}^{n}\left(\partial_{j} a_{i j}(x)\right) \partial_{i} v(t, x)
$$

and $\partial_{j} a_{i j} \in L^{\infty}(\Omega)$. Therefore

$$
\begin{aligned}
& C_{1}\left(\int_{\left(\tau_{1}, \tau_{2}\right) \times \Omega}|L v|^{2} \mathrm{e}^{2 s \varphi} \mathrm{d} x \mathrm{~d} t+\int_{\left(\tau_{1}, \tau_{2}\right) \times \Omega}|\nabla v|^{2} \mathrm{e}^{2 s \varphi} \mathrm{d} x \mathrm{~d} t\right) \\
\geq & \int_{\left(\tau_{1}, \tau_{2}\right) \times \Omega}|\widetilde{L} v|^{2} \mathrm{e}^{2 s \varphi} \mathrm{d} x \mathrm{~d} t .
\end{aligned}
$$

Hence in (2.6) with $\widetilde{L}$, we further choose $s_{0}>0$ sufficiently large and we absorb the term $C_{1} \int_{\left(\tau_{1}, \tau_{2}\right) \times \Omega}|\nabla v|^{2} \mathrm{e}^{2 s \varphi} \mathrm{d} x \mathrm{~d} t$ into the left-hand side. Moreover, fixing $\lambda$ in (2.6), we obtain (2.4).

Henceforth we set

$$
Q_{1}=\left(\tau_{1}, \tau_{2}\right) \times \Omega, \quad \Sigma_{1}=\left(\tau_{1}, \tau_{2}\right) \times \partial \Omega
$$

and

$$
a(x, \zeta, \xi) \equiv \sum_{i, j=1}^{n} a_{i j}(x) \zeta_{i} \xi_{j}, \quad \zeta=\left(\zeta_{1}, \ldots, \zeta_{n}\right), \quad \xi=\left(\xi_{1}, \ldots, \xi_{n}\right) \in \mathbb{R}^{n}, \quad(t, x) \in Q_{1}
$$

Let $w(t, x)=\mathrm{e}^{s \varphi} v(t, x)$. By (2.5) we have

$$
w\left(\tau_{1}, \cdot\right)=w\left(\tau_{2}, \cdot\right)=0 \quad \text { in } \Omega
$$

Let

$$
P w \equiv \mathrm{e}^{s \varphi} \widetilde{L} \mathrm{e}^{-s \varphi} w=\mathrm{e}^{s \varphi} \widetilde{L} v \quad \text { in } Q_{1} .
$$

It is easy to see that the operator $P$ has the form

$$
\begin{aligned}
& P w=\partial_{t} w-\sum_{i, j=1}^{n} a_{i j} \partial_{i} \partial_{j} w+2 s \lambda \varphi \sum_{i, j=1}^{n} a_{i j}\left(\partial_{i} d\right) \partial_{j} w \\
& +s \lambda^{2} \varphi w \sum_{i, j=1}^{n} a_{i j}\left(\partial_{i} d\right) \partial_{j} d-s^{2} \lambda^{2} \varphi^{2} w \sum_{i, j=1}^{n} a_{i j}\left(\partial_{i} d\right) \partial_{j} d \\
& +s \lambda \varphi w \sum_{i, j=1}^{n} a_{i j} \partial_{i} \partial_{j} d-s w \partial_{t} \varphi
\end{aligned}
$$


We set

$$
\begin{gathered}
P_{1} w+P_{2} w=P w-s \lambda^{2} \varphi w \sum_{i, j=1}^{n} a_{i j}\left(\partial_{i} d\right) \partial_{j} d \\
-s \lambda \varphi w \sum_{i, j=1}^{n} a_{i j} \partial_{i} \partial_{j} d+s w \partial_{t} \varphi \equiv f_{s} \quad \text { in } Q_{1},
\end{gathered}
$$

where

$$
\begin{gathered}
P_{1} w=-\sum_{i, j=1}^{n} a_{i j} \partial_{i} \partial_{j} w-s^{2} \lambda^{2} \varphi^{2} a(x, \nabla d, \nabla d) w \\
P_{2} w=\partial_{t} w+2 s \lambda \varphi \sum_{i, j=1}^{n} a_{i j}\left(\partial_{i} d\right) \partial_{j} w
\end{gathered}
$$

Equation (A.3) implies

$$
\left\|f_{s}\right\|_{L^{2}\left(Q_{1}\right)}^{2}=\left\|P_{1} w\right\|_{L^{2}\left(Q_{1}\right)}^{2}+\left\|P_{2} w\right\|_{L^{2}\left(Q_{1}\right)}^{2}+2\left(P_{1} w, P_{2} w\right)_{L^{2}\left(Q_{1}\right)} .
$$

By virtue of (A.4) and (A.5) we have

$$
\begin{aligned}
& \left(P_{1} w, P_{2} w\right)_{L^{2}\left(Q_{1}\right)}=\left(-\sum_{i, j=1}^{n} a_{i j} \partial_{i} \partial_{j} w-s^{2} \lambda^{2} \varphi^{2} w a(x, \nabla d, \nabla d), \partial_{t} w\right)_{L^{2}\left(Q_{1}\right)} \\
& -\int_{Q_{1}} 2 s^{3} \lambda^{3} w \varphi^{3} a(x, \nabla d, \nabla d) a(x, \nabla d, \nabla w) \mathrm{d} x \mathrm{~d} t-\int_{Q_{1}} 2 s \lambda \varphi \sum_{i, j=1}^{n} a_{i j} \partial_{i} \partial_{j} w \sum_{k, \ell=1}^{n} a_{k \ell}\left(\partial_{k} d\right) \partial_{\ell} w \mathrm{~d} x \mathrm{~d} t \\
& \equiv I_{1}+I_{2}+I_{3} .
\end{aligned}
$$

We note

$$
\nabla w=\left(\partial_{\nu} w\right) \nu \quad \text { on } \Sigma_{1},
$$

because $v \in L^{2}\left(\tau_{1}, \tau_{2} ; H^{2}(\Omega) \cap H_{0}^{1}(\Omega)\right)$ implies $\left.w\right|_{\Sigma_{1}}=0$.

Noting also that $a_{i j}=a_{j i}$ and $w\left(\tau_{1}, \cdot\right)=w\left(\tau_{2}, \cdot\right)=0$, we transform $I_{1}, I_{2}$ and $I_{3}$ by integrating by parts respectively:

$$
\begin{gathered}
I_{1}=\int_{Q_{1}}\left[\partial_{t} w \sum_{i, j=1}^{n}\left(\partial_{i} a_{i j}\right) \partial_{j} w+\sum_{i, j=1}^{n} a_{i j}\left(\partial_{j} w\right) \partial_{i} \partial_{t} w-\frac{s^{2} \lambda^{2}}{2} \varphi^{2} a(x, \nabla d, \nabla d) \partial_{t}\left(w^{2}\right)\right] \mathrm{d} x \mathrm{~d} t \\
=\int_{Q_{1}}\left[\partial_{t} w \sum_{i, j=1}^{n}\left(\partial_{i} a_{i j}\right) \partial_{j} w+w^{2} \frac{s^{2} \lambda^{2}}{2} \partial_{t}\left(\varphi^{2} a(x, \nabla d, \nabla d)\right)\right] \mathrm{d} x \mathrm{~d} t \\
I_{2}=-\int_{Q_{1}} s^{3} \lambda^{3} \varphi^{3} \sum_{i, j=1}^{n} a_{i j} a(x, \nabla d, \nabla d)\left(\partial_{i} d\right) \partial_{j}\left(w^{2}\right) \mathrm{d} x \mathrm{~d} t \\
=\int_{Q_{1}}\left[3 s^{3} \lambda^{4} w^{2} \varphi^{3} a(x, \nabla d, \nabla d)^{2}+s^{3} \lambda^{3} w^{2} \varphi^{3} \sum_{i, j=1}^{n} \partial_{j}\left(a_{i j} a(x, \nabla d, \nabla d) \partial_{i} d\right)\right] \mathrm{d} x \mathrm{~d} t
\end{gathered}
$$


and

$$
\begin{aligned}
& I_{3}=\int_{Q_{1}}-\left(\sum_{i, j=1}^{n} a_{i j} \partial_{i} \partial_{j} w\right)\left(2 s \lambda \varphi \sum_{k, \ell=1}^{n} a_{k \ell}\left(\partial_{k} d\right) \partial_{\ell} w\right) \mathrm{d} x \mathrm{~d} t \\
& =\int_{Q_{1}}\left\{\sum_{i, j=1}^{n} 2 s \lambda \varphi\left(\partial_{j} a_{i j}\right)\left(\partial_{i} w\right) \sum_{k, \ell=1}^{n} a_{k \ell}\left(\partial_{k} d\right) \partial_{\ell} w+2 s \lambda^{2} \varphi \sum_{i, j=1}^{n} a_{i j}\left(\partial_{i} w\right)\left(\partial_{j} d\right) \sum_{k, \ell=1}^{n} a_{k \ell}\left(\partial_{k} d\right) \partial_{\ell} w\right. \\
& \left.+2 s \lambda \varphi \sum_{i, j=1}^{n}\left[a_{i j} \partial_{i} w \sum_{k, \ell=1}^{n} \partial_{j}\left(a_{k \ell} \partial_{k} d\right) \partial_{\ell} w\right]+2 s \lambda \varphi \sum_{i, j=1}^{n}\left(a_{i j} \partial_{i} w \sum_{k, \ell=1}^{n} a_{k \ell}\left(\partial_{k} d\right) \partial_{j} \partial_{\ell} w\right)\right\} \mathrm{d} x \mathrm{~d} t \\
& -2 \int_{\Sigma_{1}}\left(\sum_{i, j=1}^{n} a_{i j} \nu_{j} \partial_{i} w\right)\left(s \lambda \varphi \sum_{k, \ell=1}^{n} a_{k \ell}\left(\partial_{k} d\right) \partial_{\ell} w\right) \mathrm{d} \Sigma .
\end{aligned}
$$

By using (A.8) and $a_{i j}=a_{j i}$, we can obtain

$$
\begin{aligned}
& I_{3}=\int_{Q_{1}}\left\{\sum_{i, j=1}^{n} 2 s \lambda \varphi\left(\partial_{j} a_{i j}\right) \partial_{i} w \sum_{k, \ell=1}^{n} a_{k \ell}\left(\partial_{k} d\right) \partial_{\ell} w+2 s \lambda^{2} \varphi \sum_{i, j=1}^{n} a_{i j}\left(\partial_{i} w\right)\left(\partial_{j} d\right) \sum_{k, \ell=1}^{n} a_{k \ell}\left(\partial_{k} d\right) \partial_{\ell} w\right. \\
& \left.+2 s \lambda \varphi \sum_{i, j=1}^{n}\left[a_{i j}\left(\partial_{i} w\right) \sum_{k, \ell=1}^{n} \partial_{j}\left(a_{k \ell} \partial_{k} d\right) \partial_{\ell} w\right]+s \lambda \varphi \sum_{k, \ell=1}^{n}\left[a_{k \ell} \partial_{k} d \sum_{i, j=1}^{n} a_{i j} \partial_{\ell}\left(\left(\partial_{i} w\right) \partial_{j} w\right)\right]\right\} \mathrm{d} x \mathrm{~d} t \\
& -2 s \lambda \int_{\Sigma_{1}} \varphi\left|\partial_{\nu} w\right|^{2} a(x, \nu, \nu) a(x, \nabla d, \nu) \mathrm{d} \Sigma .
\end{aligned}
$$

Integrating by parts, we have

$$
\begin{aligned}
& I_{3}=\int_{Q_{1}}\left\{\sum_{i, j=1}^{n} 2 s \lambda \varphi\left(\partial_{j} a_{i j}\right)\left(\partial_{i} w\right) \sum_{k, \ell=1}^{n} a_{k \ell}\left(\partial_{k} d\right) \partial_{\ell} w\right. \\
& +2 s \lambda^{2} \varphi a(x, \nabla d, \nabla w)^{2}+2 s \lambda \varphi \sum_{i, j=1}^{n}\left[a_{i j} \partial_{i} w \sum_{k, \ell=1}^{n} \partial_{j}\left(a_{k \ell} \partial_{k} d\right) \partial_{\ell} w\right] \\
& -s \lambda^{2} \varphi a(x, \nabla d, \nabla d) a(x, \nabla w, \nabla w)-s \lambda \varphi \sum_{k, \ell=1}^{n} \partial_{\ell}\left(a_{k \ell} \partial_{k} d\right) a(x, \nabla w, \nabla w) \\
& \left.-s \lambda \varphi \sum_{k, \ell=1}^{n}\left[a_{k \ell} \partial_{k} d \sum_{i, j=1}^{n}\left(\partial_{\ell} a_{i j}\right)\left(\partial_{i} w\right) \partial_{j} w\right]\right\} \mathrm{d} x \mathrm{~d} t \\
& -s \lambda \int_{\Sigma_{1}} \varphi\left|\partial_{\nu} w\right|^{2} a(x, \nu, \nu) a(x, \nabla d, \nu) \mathrm{d} \Sigma .
\end{aligned}
$$


By (A.9)-(A.11), we have

$$
\begin{aligned}
& \left(P_{1} w, P_{2} w\right)_{L^{2}\left(Q_{1}\right)}=\int_{Q_{1}}\left[3 s^{3} \lambda^{4} \varphi^{3} w^{2} a(x, \nabla d, \nabla d)^{2}+\left(\sum_{i, j=1}^{n}\left(\partial_{j} a_{i j}\right) \partial_{i} w\right) P_{2} w\right. \\
& \left.+2 s \lambda^{2} \varphi a(x, \nabla d, \nabla w)^{2}-s \lambda^{2} \varphi a(x, \nabla d, \nabla d) a(x, \nabla w, \nabla w)\right] \mathrm{d} x \mathrm{~d} t \\
& -s \lambda \int_{\Sigma_{1}} \varphi\left|\partial_{\nu} w\right|^{2} a(x, \nu, \nu) a(x, \nabla d, \nu) \mathrm{d} \Sigma+X_{1},
\end{aligned}
$$

where

$$
\begin{aligned}
& X_{1}=\int_{Q_{1}}\left\{w^{2} \frac{s^{2} \lambda^{2}}{2} \partial_{t}\left(\varphi^{2} a(x, \nabla d, \nabla d)\right)+s^{3} \lambda^{3} w^{2} \varphi^{3} \sum_{i, j=1}^{n} \partial_{j}\left(a_{i j} a(x, \nabla d, \nabla d) \partial_{i} d\right)\right. \\
& +2 s \lambda \varphi \sum_{i, j=1}^{n}\left[a_{i j}\left(\partial_{i} w\right) \sum_{k, \ell=1}^{n} \partial_{j}\left(a_{k \ell} \partial_{k} d\right) \partial_{\ell} w\right]-s \lambda \varphi \sum_{k, \ell=1}^{n} \partial_{\ell}\left(a_{k \ell} \partial_{k} d\right) a(x, \nabla w, \nabla w) \\
& \left.-s \lambda \varphi \sum_{k, \ell=1}^{n}\left[a_{k \ell} \partial_{k} d \sum_{i, j=1}^{n}\left(\partial_{\ell} a_{i j}\right)\left(\partial_{i} w\right) \partial_{j} w\right]\right\} \mathrm{d} x \mathrm{~d} t .
\end{aligned}
$$

Then by $a_{i j} \in C^{1}(\bar{\Omega})$, we obtain

$$
\left|X_{1}\right| \leq C_{2} \int_{Q_{1}}\left[(s \lambda \varphi+1)|\nabla w|^{2}+\left(s^{3} \lambda^{3} \varphi^{3}+s^{2} \lambda^{3} \varphi^{2}\right) w^{2}\right] \mathrm{d} x \mathrm{~d} t
$$

Multiply (A.3) by $s \lambda^{2} \varphi w a(x, \nabla d, \nabla d)$ and integrate by parts in $Q_{1}$, and we obtain

$$
\begin{aligned}
& \int_{Q_{1}} s \lambda^{2} \varphi f_{s} a(x, \nabla d, \nabla d) w \mathrm{~d} x \mathrm{~d} t \\
& =\int_{Q_{1}}\left\{s \lambda^{2} \varphi a(x, \nabla d, \nabla d) w P_{2} w-s^{3} \lambda^{4} \varphi^{3} a(x, \nabla d, \nabla d)^{2} w^{2}\right. \\
& +s \lambda^{2} \varphi a(x, \nabla w, \nabla w) a(x, \nabla d, \nabla d)+s \lambda^{3} \varphi a(x, \nabla d, \nabla d) a(x, \nabla d, \nabla w) w \\
& \left.+s \lambda^{2} \varphi w \sum_{i, j=1}^{n} \partial_{j}\left(a_{i j} a(x, \nabla d, \nabla d)\right) \partial_{i} w\right\} \mathrm{d} x \mathrm{~d} t .
\end{aligned}
$$

Consequently

$$
\begin{aligned}
& 2 s^{3} \lambda^{4} \int_{Q_{1}} \varphi^{3} a(x, \nabla d, \nabla d)^{2} w^{2} \mathrm{~d} x \mathrm{~d} t \\
& =2 \int_{Q_{1}} s \lambda^{2} \varphi a(x, \nabla w, \nabla w) a(x, \nabla d, \nabla d) \mathrm{d} x \mathrm{~d} t+2 X_{2},
\end{aligned}
$$


where

$$
\begin{aligned}
& X_{2}=\int_{Q_{1}}\left\{s \lambda^{2} \varphi w \sum_{i, j=1}^{n} \partial_{j}\left(a_{i j} a(x, \nabla d, \nabla d)\right) \partial_{i} w+s \lambda^{3} \varphi a(x, \nabla d, \nabla d) a(x, \nabla d, \nabla w) w\right. \\
& \left.+s \lambda^{2} \varphi a(x, \nabla d, \nabla d) w P_{2} w-s \lambda^{2} f_{s} \varphi a(x, \nabla d, \nabla d) w\right\} \mathrm{d} x \mathrm{~d} t .
\end{aligned}
$$

By $a_{i j} \in C^{1}(\bar{\Omega})$ and the Schwarz inequality, we obtain

$$
\begin{aligned}
& \left|X_{2}\right| \leq \frac{1}{16}\left\|P_{2} w\right\|_{L_{2}\left(Q_{1}\right)}+C_{3} \int_{Q_{1}}\left[\left(s^{2} \lambda^{4} \varphi^{2}+s^{2} \lambda^{4} \varphi\right) w^{2}+\lambda^{2} \varphi|\nabla w|^{2}\right] \mathrm{d} x \mathrm{~d} t \\
& +\frac{1}{2}\left\|f_{s}\right\|_{L^{2}\left(Q_{1}\right)}^{2} .
\end{aligned}
$$

Using $3 s^{3} \lambda^{4} \varphi^{3} w^{2} a(x, \nabla d, \nabla d)^{2}=s^{3} \lambda^{4} \varphi^{3} w^{2} a(x, \nabla d, \nabla d)^{2}+2 s^{3} \lambda^{4} \varphi^{3} w^{2} a(x, \nabla d, \nabla d)^{2}$ in (A.12) and substituting (A.14) into the above second term, we have

$$
\begin{aligned}
& \left(P_{1} w, P_{2} w\right)_{L^{2}\left(Q_{1}\right)}=\int_{Q_{1}}\left[s^{3} \lambda^{4} \varphi^{3} w^{2} a(x, \nabla d, \nabla d)^{2}+\left(\sum_{i, j=1}^{n}\left(\partial_{j} a_{i j}\right) \partial_{i} w\right) P_{2} w\right. \\
& \left.+2 s \lambda^{2} \varphi a(x, \nabla d, \nabla w)^{2}+s \lambda^{2} \varphi a(x, \nabla d, \nabla d) a(x, \nabla w, \nabla w)\right] \mathrm{d} x \mathrm{~d} t \\
& -s \lambda \int_{\Sigma_{1}} \varphi\left|\partial_{\nu} w\right|^{2} a(x, \nu, \nu) a(x, \nabla d, \nu) \mathrm{d} \Sigma+X_{1}+2 X_{2} .
\end{aligned}
$$

Therefore we see that

$$
\begin{aligned}
& 2\left(P_{1} w, P_{2} w\right)_{L^{2}\left(Q_{1}\right)} \geq \int_{Q_{1}} 2\left[s^{3} \lambda^{4} \varphi^{3} w^{2} a(x, \nabla d, \nabla d)^{2}+s \lambda^{2} \varphi a(x, \nabla d, \nabla d) a(x, \nabla w, \nabla w)\right] \mathrm{d} x \mathrm{~d} t \\
& +\int_{Q_{1}} 2\left(\frac{1}{2} P_{2} w\right)\left(2 \sum_{i, j=1}^{n}\left(\partial_{j} a_{i j}\right) \partial_{i} w\right) \mathrm{d} x \mathrm{~d} t \\
& -2 s \lambda \int_{\Sigma_{1}} \varphi\left|\partial_{\nu} w\right|^{2} a(x, \nu, \nu) a(x, \nabla d, \nu) \mathrm{d} \Sigma+2 X_{1}+4 X_{2} .
\end{aligned}
$$

Applying

$$
2\left|\frac{1}{2}\left(P_{2} w\right)\left(2 \sum_{i, j=1}^{n}\left(\partial_{j} a_{i j}\right) \partial_{i} w\right)\right| \leq \frac{1}{4}\left|P_{2} w\right|^{2}+4\left|\sum_{i, j=1}^{n}\left(\partial_{j} a_{i j}\right) \partial_{i} w\right|^{2}
$$


by virtue of $\lambda>1$, (A.6), (A.13) and (A.15), we obtain

$$
\begin{aligned}
& \left\|f_{s}\right\|_{L^{2}\left(Q_{1}\right)}^{2}=\left\|P_{1} w\right\|_{L^{2}\left(Q_{1}\right)}^{2}+\left\|P_{2} w\right\|_{L^{2}\left(Q_{1}\right)}^{2}+2\left(P_{1} w, P_{2} w\right)_{L^{2}\left(Q_{1}\right)} \\
& \geq\left\|P_{1} w\right\|_{L^{2}\left(Q_{1}\right)}^{2}+\frac{1}{2}\left\|P_{2} w\right\|_{L^{2}\left(Q_{1}\right)}^{2} \\
& +\int_{Q_{1}} 2\left[s^{3} \lambda^{4} \varphi^{3} w^{2} a(x, \nabla d, \nabla d)^{2}+s \lambda^{2} \varphi a(x, \nabla d, \nabla d) a(x, \nabla w, \nabla w)\right] \mathrm{d} x \mathrm{~d} t \\
& -C_{4} \int_{Q_{1}}\left[\left(\lambda^{2} \varphi+s \lambda \varphi+1\right)|\nabla w|^{2}+\left(s^{3} \lambda^{3} \varphi^{3}+s^{2} \lambda^{4} \varphi^{2}+s^{2} \lambda^{4} \varphi\right) w^{2}\right] \mathrm{d} x \mathrm{~d} t \\
& -2\left\|f_{s}\right\|_{L^{2}\left(Q_{1}\right)}^{2}-2 s \lambda \int_{\Sigma_{1}} \varphi\left|\partial_{\nu} w\right|^{2} a(x, \nu, \nu) a(x, \nabla d, \nu) \mathrm{d} \Sigma .
\end{aligned}
$$

Since $d \in C^{2}(\bar{\Omega})$ satisfies $|\nabla d(x)|>0, x \in \bar{\Omega}$, by (1.4) we can obtain

$$
\begin{aligned}
& \left\|f_{s}\right\|_{L^{2}\left(Q_{1}\right)}^{2} \geq \frac{1}{3}\left\|P_{1} w\right\|_{L^{2}\left(Q_{1}\right)}^{2}+\frac{1}{6}\left\|P_{2} w\right\|_{L^{2}\left(Q_{1}\right)}^{2} \\
& +C_{5} \int_{Q_{1}}\left(s^{3} \lambda^{4} \varphi^{3} w^{2}+s \lambda^{2} \varphi|\nabla w|^{2}\right) \mathrm{d} x \mathrm{~d} t \\
& -C_{6} \int_{Q_{1}}\left[\left(\lambda^{2} \varphi+s \lambda \varphi+1\right)|\nabla w|^{2}+\left(s^{3} \lambda^{3} \varphi^{3}+s^{2} \lambda^{4} \varphi^{2}+s^{2} \lambda^{4} \varphi\right) w^{2}\right] \mathrm{d} x \mathrm{~d} t \\
& -\frac{2}{3} s \lambda \int_{\Sigma_{1}} \varphi\left|\partial_{\nu} w\right|^{2} a(x, \nu, \nu) a(x, \nabla d, \nu) \mathrm{d} \Sigma .
\end{aligned}
$$

In terms of the definition of $f_{s}$ in (A.3), we have

$$
\left\|f_{s}\right\|_{L^{2}\left(Q_{1}\right)}^{2} \leq C_{7} \int_{Q_{1}}\left(s^{2} \lambda^{4} \varphi^{2} w^{2}+|P w|^{2}\right) \mathrm{d} x \mathrm{~d} t .
$$

Therefore, using also (2.2), we obtain

$$
\begin{aligned}
& C_{8}\|P w\|_{L^{2}\left(Q_{1}\right)}^{2}+C_{8} s \lambda \int_{\tau_{1}}^{\tau_{2}} \int_{\Gamma_{0}} \varphi\left|\partial_{\nu} w\right|^{2} \mathrm{~d} \Sigma \\
& \geq \frac{1}{3}\left\|P_{1} w\right\|_{L^{2}\left(Q_{1}\right)}^{2}+\frac{1}{6}\left\|P_{2} w\right\|_{L^{2}\left(Q_{1}\right)}^{2}+\int_{Q_{1}}\left(C_{5} s^{3} \lambda^{4} \varphi^{3}-C_{8} s^{3} \lambda^{3} \varphi^{3}-C_{8} s^{2} \lambda^{4} \varphi^{2}-C_{8} s^{2} \lambda^{4} \varphi\right) w^{2} \mathrm{~d} x \mathrm{~d} t \\
& +\int_{Q_{1}}\left(C_{5} s \lambda^{2} \varphi-C_{8} s \lambda \varphi-C_{8} \lambda^{2} \varphi-C_{8}\right)|\nabla w|^{2} \mathrm{~d} x \mathrm{~d} t .
\end{aligned}
$$

Noting that $\varphi \geq 1$ on $\overline{Q_{1}}$, we can find constants $\lambda_{0}>0$ and $s_{0}>0$ which continuously depend on $\sum_{i, j=1}^{n}\left\|a_{i j}\right\|_{C^{1}(\bar{\Omega})}, \tau_{1}, \tau_{2}, \Omega, r$ such that

$$
\begin{aligned}
& C_{9} s \lambda \int_{\tau_{1}}^{\tau_{2}} \int_{\Gamma_{0}} \varphi\left|\partial_{\nu} w\right|^{2} \mathrm{~d} \Sigma+C_{9}\|P w\|_{L^{2}\left(Q_{1}\right)}^{2} \geq\left\|P_{1} w\right\|_{L^{2}\left(Q_{1}\right)}^{2}+\left\|P_{2} w\right\|_{L^{2}\left(Q_{1}\right)}^{2} \\
& +\int_{Q_{1}}\left(s^{3} \lambda^{4} \varphi^{3} w^{2}+s \lambda^{2} \varphi|\nabla w|^{2}\right) \mathrm{d} x \mathrm{~d} t
\end{aligned}
$$


for all $s>s_{0}$ and $\lambda>\lambda_{0}$. By (A.4) and (A.5), we have

$$
\left|\left(P_{1} w\right)(x, t)\right|^{2} \geq C_{10}\left|\sum_{i, j=1}^{n} a_{i j} \partial_{i} \partial_{j} w\right|^{2}-C_{11} s^{4} \lambda^{4} \varphi^{4} w^{2}
$$

and

$$
\left|\left(P_{2} w\right)(x, t)\right|^{2} \geq C_{10}\left|\partial_{t} w\right|^{2}-C_{11} s^{2} \lambda^{2} \varphi^{2}|\nabla w|^{2}
$$

so that

$$
\begin{aligned}
& \int_{Q_{1}}\left\{\frac{1}{s \varphi}\left(\left|\partial_{t} w\right|^{2}+\left|\sum_{i, j=1}^{n} a_{i j} \partial_{i} \partial_{j} w\right|^{2}\right)+s \lambda^{2} \varphi|\nabla w|^{2}+s^{3} \lambda^{4} \varphi^{3} w^{2}\right\} \mathrm{d} x \mathrm{~d} t \\
& \leq C_{12} \int_{Q_{1}}|P w|^{2} \mathrm{~d} x \mathrm{~d} t+C_{12} s \lambda \int_{\tau_{1}}^{\tau_{2}} \int_{\Gamma_{0}} \varphi\left|\partial_{\nu} w\right|^{2} \mathrm{~d} \Sigma
\end{aligned}
$$

for all $s>s_{0}$ and $\lambda>\lambda_{0}$.

Moreover we have

$$
\begin{aligned}
& \partial_{i} \partial_{j}\left(\frac{w}{\sqrt{\varphi}}\right)=\frac{\partial_{i} \partial_{j} w}{\sqrt{\varphi}}-\frac{\partial_{i} \partial_{j} \varphi}{2 \varphi^{\frac{3}{2}}} w \\
- & \frac{1}{2 \varphi^{\frac{3}{2}}}\left\{\left(\partial_{j} w\right)\left(\partial_{i} \varphi\right)+\left(\partial_{i} w\right)\left(\partial_{j} \varphi\right)\right\}+\frac{3}{4 \varphi^{\frac{5}{2}}}\left(\partial_{i} \varphi\right)\left(\partial_{j} \varphi\right) w, \quad 1 \leq i, j \leq n,
\end{aligned}
$$

and

$$
\begin{aligned}
& \sum_{i, j=1}^{n} a_{i j} \partial_{i} \partial_{j}\left(\frac{w}{\sqrt{\varphi}}\right) \\
= & \frac{g}{\sqrt{\varphi}}-\frac{\sum_{i, j=1}^{n} a_{i j} \partial_{i} \partial_{j} \varphi}{2 \varphi^{\frac{3}{2}}} w+\frac{3}{4 \varphi^{\frac{5}{2}}} w \sum_{i, j=1}^{n} a_{i j}\left(\partial_{i} \varphi\right)\left(\partial_{j} \varphi\right) \\
- & \frac{1}{\varphi^{\frac{3}{2}}} \sum_{i, j=1}^{n} a_{i j}\left(\partial_{i} w\right)\left(\partial_{j} \varphi\right)
\end{aligned}
$$

where we set $g=\sum_{i, j=1}^{n} a_{i j} \partial_{i} \partial_{j} w$. Since $w(t, \cdot) \in H_{0}^{1}(\Omega)$ for almost all $t \in\left[\tau_{1}, \tau_{2}\right]$, we apply a usual a priori estimate for the Dirichlet problem for the elliptic equation (e.g., [16]), so that

$$
\begin{aligned}
& \int_{\Omega} \sum_{i, j=1}^{n}\left|\partial_{i} \partial_{j}\left(\frac{w}{\sqrt{\varphi}}\right)\right|^{2}(t, x) \mathrm{d} x \leq C_{13} \int_{\Omega} \frac{g(t, x)^{2}}{\varphi} \mathrm{d} x+C_{13} \int_{\Omega} \frac{\left|\sum_{i, j=1}^{n} a_{i j} \partial_{i} \partial_{j} \varphi\right|^{2}}{\varphi^{3}}|w(t, x)|^{2} \mathrm{~d} x \\
& +C_{13} \int_{\Omega} \frac{w(t, x)^{2}}{\varphi^{5}}\left|\sum_{i, j=1}^{n} a_{i j}\left(\partial_{i} \varphi\right)\left(\partial_{j} \varphi\right)\right|^{2} \mathrm{~d} x \\
& +C_{13} \int_{\Omega} \frac{1}{\varphi^{3}}\left|\sum_{i, j=1}^{n} a_{i j}\left(\partial_{i} w\right) \partial_{j} \varphi\right|^{2} \mathrm{~d} x .
\end{aligned}
$$


On the other hand, (A.17) yields

$$
\begin{aligned}
& \int_{\Omega} \frac{1}{\varphi}\left|\partial_{i} \partial_{j} w(t, x)\right|^{2} \mathrm{~d} x \\
& \leq C_{14} \int_{\Omega}\left\{\left|\partial_{i} \partial_{j}\left(\frac{w}{\sqrt{\varphi}}\right)\right|^{2}+\frac{\left|\partial_{i} \partial_{j} \varphi\right|^{2}}{\varphi^{3}} w^{2}+\frac{1}{\varphi^{3}}\left(\left|\partial_{j} w\right|^{2}\left|\partial_{i} \varphi\right|^{2}+\left|\partial_{i} w\right|^{2}\left|\partial_{j} \varphi\right|^{2}\right)\right. \\
& \left.+\frac{1}{\varphi^{5}}\left|\partial_{i} \varphi\right|^{2}\left|\partial_{j} \varphi\right|^{2} w^{2}\right\}(t, x) \mathrm{d} x
\end{aligned}
$$

Since $\partial_{i} \varphi=\lambda\left(\partial_{i} d\right) \varphi$ and $\partial_{i} \partial_{j} \varphi=\lambda\left(\partial_{i} \partial_{j} d\right) \varphi+\lambda^{2}\left(\partial_{i} d\right)\left(\partial_{j} d\right) \varphi$, we see by $\lambda>1$ that

$$
\begin{aligned}
& \left|\partial_{i} \varphi(t, x)\right| \leq C_{15} \lambda \varphi(t, x), \\
& \left|\partial_{i} \partial_{j} \varphi(t, x)\right| \leq C_{15} \lambda^{2} \varphi(t, x), \quad 1 \leq i, j \leq n,(t, x) \in \overline{Q_{1}} .
\end{aligned}
$$

Hence, $\varphi \geq 1$, (A.18) and (A.19) yield

$$
\sum_{i, j=1}^{n} \int_{\Omega} \frac{1}{\varphi(t, x)}\left|\partial_{i} \partial_{j} w(t, x)\right|^{2} \mathrm{~d} x \leq C_{16} \int_{\Omega} \frac{g^{2}(t, x)}{\varphi(t, x)}+C_{16} \int_{\Omega}\left(\lambda^{4} w^{2}+\lambda^{2}|\nabla w|^{2}\right)(t, x) \mathrm{d} x .
$$

With (A.16), we obtain

$$
\begin{aligned}
& \int_{Q_{1}}\left\{\frac{1}{s \varphi}\left(\left|\partial_{t} w\right|^{2}+\sum_{i, j=1}^{n}\left|\partial_{i} \partial_{j} w\right|^{2}\right)+s \lambda^{2} \varphi|\nabla w|^{2}+s^{3} \lambda^{4} \varphi^{3} w^{2}\right\} \mathrm{d} x \mathrm{~d} t \\
& \leq C_{17} \int_{Q_{1}}|P w|^{2} \mathrm{~d} x \mathrm{~d} t+C_{17} s \lambda \int_{\tau_{1}}^{\tau_{2}} \int_{\Gamma_{0}} \varphi\left|\partial_{\nu} w\right|^{2} \mathrm{~d} \Sigma
\end{aligned}
$$

for all $s>s_{0}$ and $\lambda>\lambda_{0}$. Substituting $w=\mathrm{e}^{s \varphi} v$ and noting $\left.v\right|_{\Sigma_{1}}=0$ and (A.20), we can complete the proof of $(2.6)$.

In (2.6), fixing $\lambda>\lambda_{0}$ and replacing $\mathrm{e}^{\lambda M_{1}} s$ by $s$, we can derive (2.4) from (2.6). Thus the proof of Theorem 2.1 is completed.

\section{Appendix B. Proof of (1.6) AND (1.10)}

For $\left\{a_{i j}\right\} \in \mathcal{U}$, we recall that the operator $A$ in $L^{2}(\Omega)$ is defined by (1.9), and that (4.1) holds.

Moreover the fractional power $A^{\gamma}, \gamma \in \mathbb{R}$ is defined (e.g., [37]). Applying the interpolation theorem (e.g., [35]) in (4.1), we see that

$$
C_{1}^{-1}\left\|A^{\frac{m_{0}}{2}} u\right\| \leq\|u\|_{H^{m_{0}}(\Omega)} \leq C_{1}\left\|A^{\frac{m_{0}}{2}} u\right\|, \quad m_{0}=0,1,2, \ldots, 2\left[\frac{m+1}{2}\right], u \in \mathcal{D}\left(A^{\frac{m_{0}}{2}}\right) .
$$

On the other hand, $-A$ generates an analytic semigroup in $L^{2}(\Omega)($ e.g., [37]) and we have

$$
y(t) \equiv y\left(\left\{a_{i j}\right\}, h, \mu\right)(t, \cdot)=\mathrm{e}^{-t A} \mu+\int_{0}^{t} \mathrm{e}^{-s A} h(t-s) \mathrm{d} s, \quad 0<t<T .
$$

Here and henceforth we regard $h(t)=h(t, \cdot)$ as an element in $L^{2}\left(0, T ; L^{2}(\Omega)\right)$. Therefore by $h \in C_{0}^{\infty}((0, T) \times \omega)$, we have

$$
\partial_{t}^{\ell} y(t)=(-A)^{\ell} \mathrm{e}^{-t A} \mu+\int_{0}^{t} \mathrm{e}^{-s A} \partial_{t}^{\ell} h(t-s) \mathrm{d} s, \quad 0<t<T
$$


Furthermore $h \in C_{0}^{\infty}((0, T) \times \omega)$ yields $\left\|A^{\frac{m_{0}}{2}} \partial_{t}^{\ell} h(t)\right\| \leq C_{2}\left\|\partial_{t}^{\ell} h(t)\right\|_{H^{m_{0}}(\omega)}$ by (B.1). On the other hand, $\left\|A^{\ell} \mathrm{e}^{-t A}\right\| \leq \frac{C_{3}}{t^{\ell}}$ for $t>0$ (e.g., Sect. 2.6 in [37]), and by the proof in [37], we see that the constant $C_{3}>0$ can be chosen uniformly in $\left\{a_{i j}\right\} \in \mathcal{U}$. Consequently we obtain

$$
\begin{aligned}
& \left\|A^{\frac{m_{0}}{2}} \partial_{t}^{\ell} y(t)\right\| \leq \frac{C_{3}}{\tau_{1}^{\ell+\frac{m_{0}}{2}}}\|\mu\|+C_{3} \int_{0}^{t}\left\|A^{\frac{m_{0}}{2}} \partial_{t}^{\ell} h(t-s)\right\| \mathrm{d} s \\
& \leq\left(\frac{C_{3}}{\tau_{1}^{\ell+\frac{m_{0}}{2}}}+C_{3}\right)\left(\|\mu\|+\|h\|_{W^{\ell, 1}\left(0, T ; H^{m_{0}}(\omega)\right)}\right), \quad \tau_{1} \leq t \leq \tau_{2} .
\end{aligned}
$$

Thus, in terms of (B.1), the proof of (1.6) is completed.

Next we prove $(1.10)$. We see that $H^{m}(\Omega) \subset C^{2}(\bar{\Omega})$ from the Sobolev embedding theorem (e.g., [1,35]). Similarly to (B.2), in terms of (B.1) we have

$$
\begin{aligned}
& \left\|y\left(\left\{a_{i j}\right\}, h, 0\right)\right\|_{C[0, T] ; C^{2}(\bar{\Omega})} \leq C_{4}\left\|y\left(\left\{a_{i j}\right\}, h, 0\right)\right\|_{C[0, T] ; H^{m}(\Omega)} \\
& \leq C_{5} \int_{0}^{t}\left\|A^{\frac{m}{2}} \mathrm{e}^{-s A} h(t-s)\right\| \mathrm{d} s=C_{5} \int_{0}^{t}\left\|\mathrm{e}^{-s A} A^{\frac{m}{2}} h(t-s)\right\| \mathrm{d} s \leq C_{6} \int_{0}^{t}\|h(t-s)\|_{H^{m}(\Omega)} \mathrm{d} s .
\end{aligned}
$$

Thus the proof of (1.10) is completed.

\section{Appendix C. Proof of Lemma 2.1}

In Chae, Imanuvilov and Kim [9], Fursikov and Imanuvilov [15], the following lemma is proved. See also Imanuvilov [19].

Lemma C.1. Let $\widetilde{\Omega} \subset \mathbb{R}^{n}$ be a bounded domain whose boundary $\partial \widetilde{\Omega}$ is of class $C^{2}$ and $\omega \subset \widetilde{\Omega}$ be a subdomain such that $\bar{\omega} \subset \widetilde{\Omega}$. Then there exists a function $d \in C^{2}(\overline{\widetilde{\Omega}})$ such that

$$
d(x)>0, x \in \widetilde{\Omega},\left.\quad d\right|_{\partial \widetilde{\Omega}}=0, \quad|\nabla d(x)|>0, x \in \overline{\widetilde{\Omega} \backslash \omega_{1}} .
$$

Now we proceed to the proof of Lemma 2.1. Let us enlarge the domain $\Omega$ to a domain $\widetilde{\Omega}$ which has the following properties:

$$
\Omega \subset \widetilde{\Omega} \quad \partial \Omega \backslash \Gamma_{0} \subset \partial \widetilde{\Omega} \quad \operatorname{Int}(\widetilde{\Omega} \backslash \Omega) \neq \emptyset .
$$

Choose a subdomain $\omega$ such that $\bar{\omega} \subset \operatorname{Int}(\widetilde{\Omega} \backslash \Omega)$. Thus, by Lemma C.1, there exists a function $d(x)$ in $\widetilde{\Omega}$ which satisfies $d(x)>0, x \in \widetilde{\Omega},\left.d\right|_{\partial \widetilde{\Omega}}=0$ and $|\nabla d(x)|>0, x \in \overline{\widetilde{\Omega}} \backslash \omega$. Therefore $d(x)>0, x \in \Omega$ and $|\nabla d(x)|>0, x \in \bar{\Omega}$. Finally, we have to verify $\sum_{i, j=1}^{n} a_{i j}(x) \partial_{i} d(x) \nu_{j}(x) \leq 0, x \in \partial \Omega \backslash \Gamma_{0}$. Since $\partial \Omega \backslash \Gamma_{0} \subset \partial \widetilde{\Omega}$, we have $\left.d\right|_{\partial \Omega \backslash \Gamma_{0}}=0$ from $\left.d\right|_{\partial \tilde{\Omega}}=0$, which implies $\nabla d(x)=\partial_{\nu} d(x) \nu(x), x \in \partial \Omega \backslash \Gamma_{0}$, that is, $\nabla d$ is parallel to $\nu$ on $\partial \Omega \backslash \Gamma_{0}$. Therefore, by $d>0$ in $\Omega$, we have $\nabla d(x)=-|\nabla d(x)| \nu(x), x \in \partial \Omega \backslash \Gamma_{0}$. By $|\nabla d(x)|>0$ on $\bar{\Omega}$, we obtain $\nu(x)=-\frac{\nabla d(x)}{|\nabla d(x)|}, x \in \partial \Omega \backslash \Gamma_{0}$. Therefore by (1.4) we see

$$
\sum_{i, j=1}^{n} a_{i j}(x) \partial_{i} d(x) \nu_{j}(x)=-\frac{\sum_{i, j=1}^{n} a_{i j}(x) \partial_{i} d(x) \partial_{j} d(x)}{|\nabla d(x)|} \leq 0, \quad x \in \partial \Omega \backslash \Gamma_{0} .
$$

Thus the proof of Lemma 2.1 is completed. 
Acknowledgements. The first author is partially supported by the Japanese Government Scholarship. The second author is partially supported by Grant 15340027 from the Japan Society for the Promotion of Science and Grant 17654019 from the Ministry of Education, Cultures, Sports and Technology. The authors sincerely thank the anonymous referees for their thorough reading and many invaluable comments.

\section{REFERENCES}

[1] R.A. Adams, Sobolev Spaces. Academic Press, New York (1975).

[2] K.A. Ames and B. Straughan, Non-standard and Improperly Posed Problems. Academic Press, San Diego (1997).

[3] L. Baudouin and J.-P. Puel, Uniqueness and stability in an inverse problem for the Schrödinger equation. Inverse Probl. 18 (2002) 1537-1554.

[4] M. Bellassoued, Global logarithmic stability in inverse hyperbolic problem by arbitrary boundary observation. Inverse Probl. 20 (2004) 1033-1052.

[5] M. Bellassoued and M. Yamamoto, Logarithmic stability in determination of a coefficient in an acoustic equation by arbitrary boundary observation. J. Math. Pures Appl. 85 (2006) 193-224.

[6] H. Brezis, Analyse Fonctionnelle. Masson, Paris (1983).

[7] A.L. Bukhgeim, Introduction to the Theory of Inverse Probl. VSP, Utrecht (2000).

[8] A.L. Bukhgeim and M.V. Klibanov, Global uniqueness of a class of multidimensional inverse problems. Soviet Math. Dokl. 24 (1981) 244-247.

[9] D. Chae, O.Yu. Imanuvilov and S.M. Kim, Exact controllability for semilinear parabolic equations with Neumann boundary conditions. J. Dyn. Contr. Syst. 2 (1996) 449-483.

[10] J. Cheng and M. Yamamoto, One new strategy for a priori choice of regularizing parameters in Tikhonov's regularization. Inverse Probl. 16 (2000) L31-L38.

[11] P.G. Danilaev, Coefficient Inverse Problems for Parabolic Type Equations and Their Application. VSP, Utrecht (2001).

[12] A. Elayyan and V. Isakov, On uniqueness of recovery of the discontinuous conductivity coefficient of a parabolic equation. SIAM J. Math. Anal. 28 (1997) 49-59.

[13] M.M. Eller and V. Isakov, Carleman estimates with two large parameters and applications. Contemp. Math. 268 (2000) $117-136$.

[14] C. Fabre, J.-P. Puel and E. Zuazua, Approximate controllability of the semilinear heat equation. Proc. Royal Soc. Edinburgh 125A (1995) 31-61.

[15] A.V. Fursikov and O.Yu. Imanuvilov, Controllability of Evolution Equations, in Lecture Notes Series 34, Seoul National University, Seoul, South Korea (1996).

[16] D. Gilbarg and N.S. Trudinger, Elliptic Partial Differential Equations of Second Order. Springer-Verlag, Berlin (2001).

[17] R. Glowinski and J.L. Lions, Exact and approximate controllability for distributed parameter systems. Acta Numer. 3 (1994) 269-378.

[18] L. Hörmander, Linear Partial Differential Operators. Springer-Verlag, Berlin (1963).

[19] O.Yu. Imanuvilov, Controllability of parabolic equations. Sb. Math. 186 (1995) 879-900.

[20] O.Yu. Imanuvilov and M. Yamamoto, Lipschitz stability in inverse parabolic problems by the Carleman estimate. Inverse Probl. 14 (1998) 1229-1245.

[21] O.Yu. Imanuvilov and M. Yamamoto, Global Lipschitz stability in an inverse hyperbolic problem by interior observations. Inverse Probl. 17 (2001) 717-728.

[22] O.Yu. Imanuvilov and M. Yamamoto, Carleman estimate for a parabolic equation in a Sobolev space of negative order and its applications, in Control of Nonlinear Distributed Parameter Systems, Marcel Dekker, New York (2001) 113-137.

[23] O.Yu. Imanuvilov and M. Yamamoto, Determination of a coefficient in an acoustic equation with a single measurement. Inverse Probl. 19 (2003) 151-171.

[24] O.Yu. Imanuvilov and M. Yamamoto, Carleman inequalities for parabolic equations in Sobolev spaces of negative order and exact controllability for semilinear parabolic equations. Publ. RIMS Kyoto Univ. 39 (2003) 227-274.

[25] V. Isakov, Inverse Problems for Partial Differential Equations. Springer-Verlag, Berlin (1998), (2005).

[26] V. Isakov and S. Kindermann, Identification of the diffusion coefficient in a one-dimensional parabolic equation. Inverse Probl. 16 (2000) 665-680.

[27] M. Ivanchov, Inverse Problems for Equations of Parabolic Type. VNTL Publishers, Lviv, Ukraine (2003).

[28] A. Khal̆darov, Carleman estimates and inverse problems for second order hyperbolic equations. Math. USSR Sbornik 58 (1987) $267-277$.

[29] M.V. Klibanov, Inverse problems in the "large" and Carleman bounds. Diff. Equ. 20 (1984) 755-760.

[30] M.V. Klibanov, Inverse problems and Carleman estimates. Inverse Probl. 8 (1992) 575-596.

[31] M.V. Klibanov, Estimates of initial conditions of parabolic equations and inequalities via lateral Cauchy data. Inverse Probl. 22 (2006) 495-514.

[32] M.V. Klibanov and A.A. Timonov, Carleman Estimates for Coefficient Inverse Problems and Numerical Applications. VSP, Utrecht (2004). 
[33] M.V. Klibanov and M. Yamamoto, Lipschitz stability of an inverse problem for an accoustic equation. Appl. Anal. 85 (2006) $515-538$.

[34] M.M. Lavrent'ev, V.G. Romanov and Shishat-skiı̌, Ill-posed Problems of Mathematical Physics and Analysis. American Mathematical Society Providence, Rhode Island (1986).

[35] J.L. Lions and E. Magenes, Non-homogeneous Boundary Value Problems and Applications. Springer-Verlag, Berlin (1972).

[36] L.E. Payne, Improperly Posed Problems in Partial Differential Equations. SIAM, Philadelphia (1975).

[37] A. Pazy, Semigroups of Linear Operators and Applications to Partial Differential Equations. Springer-Verlag, New York (1983).

[38] J.C. Saut and B. Scheurer, Unique continuation for some evolution equations. J. Diff. Eq. 66 (1987) 118-139.

[39] E.J.P.G. Schmidt and N. Weck, On the boundary behavior of solutions to elliptic and parabolic equations - with applications to boundary control for parabolic equations. SIAM J. Contr. Opt. 16 (1978) 593-598.

[40] M. Yamamoto, Uniqueness and stability in multidimensional hyperbolic inverse problems. J. Math. Pures Appl. 78 (1999) 65-98.

[41] M. Yamamoto and J. Zou, Simultaneous reconstruction of the initial temperature and heat radiative coefficient. Inverse Probl. 17 (2001) 1181-1202. 